\title{
Dark matter search in nucleon, pion, and electron channels from a proton beam dump with MiniBooNE
}

A. A. Aguilar-Arevalo, ${ }^{1}$ M. Backfish, ${ }^{2}$ A. Bashyal, ${ }^{3}$ B. Batell, ${ }^{4}$ B. C. Brown, ${ }^{2}$ R. Carr, ${ }^{5}$ A. Chatterjee, ${ }^{3}$ R. L. Cooper, ${ }^{6,7}$ P. deNiverville, ${ }^{8}$ R. Dharmapalan, ${ }^{9}$ Z. Djurcic, ${ }^{9}$ R. Ford, ${ }^{2}$ F. G. Garcia, ${ }^{2}$ G. T. Garvey, ${ }_{10}$ J. Grange, ${ }^{9,11}$ J. A. Green, ${ }^{10}$ E.-C. Huang, ${ }^{10}$ W. Huelsnitz, ${ }^{10}$ I. L. de Icaza Astiz, ${ }^{1}$ G. Karagiorgi,${ }^{5}$ T. Katori, ${ }^{12}$ W. Ketchum, ${ }^{10}$ T. Kobilarcik, ${ }^{2}$ Q. Liu, ${ }^{10}$ W. C. Louis, ${ }^{10}$ W. Marsh,${ }^{2}$ C. D. Moore,${ }^{2}$ G. B. Mills,${ }^{10,}{ }^{*}$ J. Mirabal,${ }^{10}$ P. Nienaber, ${ }^{13}$ Z. Pavlovic, ${ }^{10}$ D. Perevalov, ${ }^{2}$ H. Ray, ${ }^{11}$ B. P. Roe, ${ }^{14}$ M. H. Shaevitz, ${ }^{5}$ S. Shahsavarani, ${ }^{3}$ I. Stancu, ${ }^{15}$ R. Tayloe,${ }^{6}$ C. Taylor, ${ }^{10}$ R. T. Thornton, ${ }^{10}$ R. G. Van de Water, ${ }^{10}$ W. Wester, ${ }^{2}$ D. H. White, ${ }^{13,10,{ }^{*}}$ and J. $\mathrm{Yu}^{3}$

(The MiniBooNE-DM Collaboration)

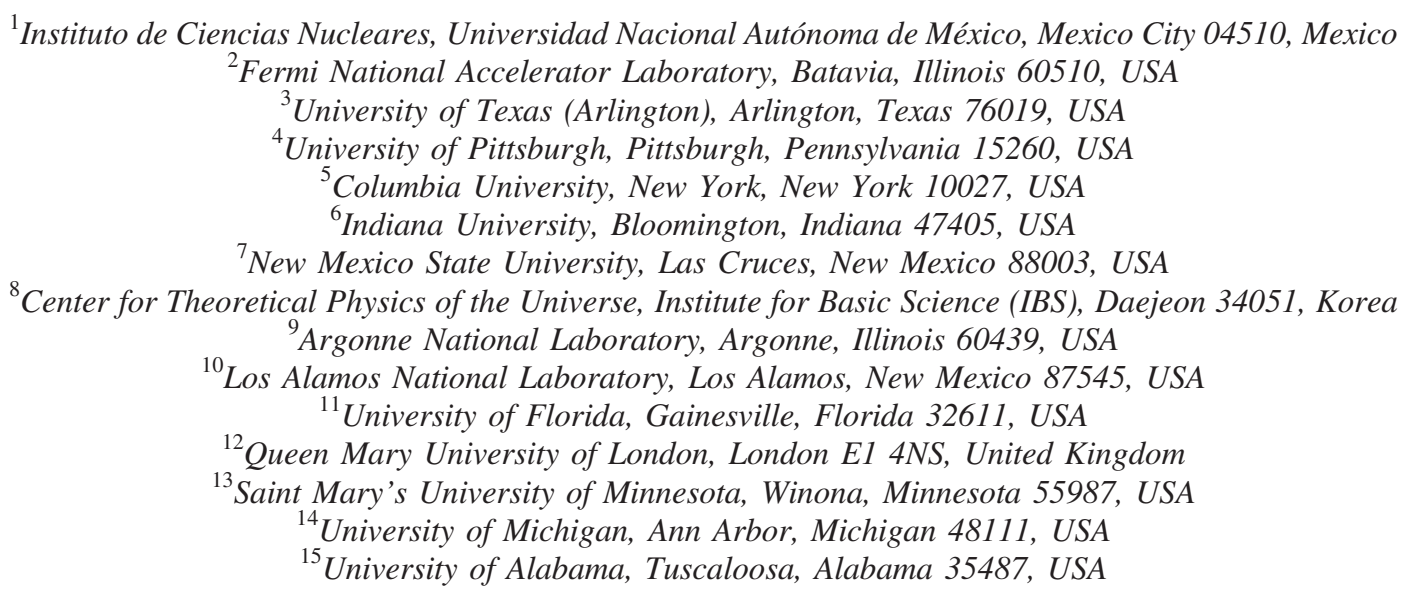

(Received 17 July 2018; published 12 December 2018)

A search for sub-GeV dark matter produced from collisions of the Fermilab $8 \mathrm{GeV}$ Booster protons with a steel beam dump was performed by the MiniBooNE-DM Collaboration using data from $1.86 \times 10^{20}$ protons on target in a dedicated run. The MiniBooNE detector, consisting of 818 tons of mineral oil and located 490 meters downstream of the beam dump, is sensitive to a variety of dark matter initiated scattering reactions. Three dark matter interactions are considered for this analysis: elastic scattering off nucleons, inelastic neutral pion production, and elastic scattering off electrons. Multiple data sets were used to constrain flux and systematic errors, and time-of-flight information was employed to increase sensitivity to higher dark matter masses. No excess from the background predictions was observed, and $90 \%$ confidence level limits were set on the vector portal and leptophobic dark matter models. New parameter space is excluded in the vector portal dark matter model with a dark matter mass between 5 and $50 \mathrm{MeV}^{-2}$. The reduced neutrino flux allowed to test if the MiniBooNE neutrino excess scales with the production of neutrinos. No excess of neutrino oscillation events were measured ruling out models that scale solely by number of protons on target independent of beam configuration at $4.6 \sigma$.

DOI: 10.1103/PhysRevD.98.112004

\section{*Deceased.}

Published by the American Physical Society under the terms of the Creative Commons Attribution 4.0 International license. Further distribution of this work must maintain attribution to the author(s) and the published article's title, journal citation, and DOI. Funded by SCOAP ${ }^{3}$.

\section{INTRODUCTION}

A wide variety of astrophysical and cosmological observations present strong evidence for the existence of dark matter, and a diverse experimental program has developed over the past few decades to search for its nongravitational interactions. In the context of the popular weak scale "WIMP" dark matter scenarios, impressive coverage has 
been achieved through several experimental and observational approaches, including direct searches for dark matter scattering with nuclei, indirect searches for dark matter annihilation in the Galaxy and beyond, and high-energy collider searches for missing energy. However, these traditional search strategies are often less sensitive to light dark matter candidates with masses below a few $\mathrm{GeV} c^{-2}$, and it is thus important to consider alternative experimental approaches to dark matter detection in this regime.

In this context, there has been a growing realization that fixed-target experiments can provide significant and complementary sensitivity to sub-GeV dark matter that couples to ordinary matter through a light mediator [1-19]. In this approach, a relativistic flux of dark matter particles is produced out of proton (or electron) collisions with a fixed target, followed by the detection of the dark matter through its scattering in a detector placed downstream of the target. This approach was successfully employed at Fermilab with the MiniBooNE detector, setting new limits on sub-GeV dark matter in the neutral-current (quasi)elastic nucleon scattering with no pion in the final state (NCE) [20].

The MiniBooNE experiment was designed to study short-baseline neutrino oscillations [21]. In the normal neutrino or antineutrino running modes, charged pions $\pi^{ \pm}$are produced in the collisions of the proton beam with a beryllium target and subsequently decay in flight to neutrinos in the decay volume immediately following the target, as shown in Fig. 1. This results in a large flux of neutrinos at the MiniBooNE detector, which is a background to the dark matter neutral-current-like scattering signature. Instead, in the beam-dump running mode, the proton beam is steered past the beryllium target and directed onto the steel absorber at the end of the decay volume, which significantly reduces the neutrino flux and increases sensitivity to a potential dark matter signal. A dedicated run in beam-dump mode was carried out from November 2013 to September 2014 collecting $1.86 \times 10^{20}$ protons on target (POT). Besides the capability of running in beam-dump mode, MiniBooNE has several advantages which make this search feasible, including a detailed understanding of detector response and standard background processes gained through over a decade of operation, and robust and well-tested particle identification techniques.
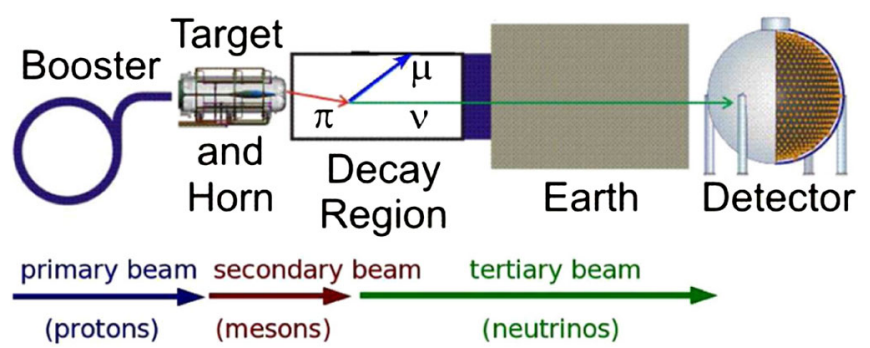

FIG. 1. The production of neutrinos in the Booster Neutrino Beamline in on-target running [22].
The results presented here improve upon those in Ref. [20] by including two additional dark matter interaction channels in two separate analyses. The first was a combined NCE and neutral-current pion production through delta resonant decay $(\mathrm{NC} \pi)$ fit to search for dark matter interaction with nucleons, and the second was dark matter elastically scattering off electrons. A "time-offlight" observable was also added to both analyses to increase sensitivity to heavier dark matter masses. No significant excess is observed in either analysis, and 90\% confidence level limits are derived for vector portal and leptophobic dark matter models. MiniBooNE excludes new parameter space in the vector portal dark matter model.

Results from applying the neutrino oscillation cuts are also presented. With the reduction of the neutrino flux, a test was preformed to determine if the neutrino oscillation excess [21,23] comes from a process that scales with neutrino production or a process that would scale solely on the number of POT.

The following section provides an overview of the theoretical aspects of sub-GeV dark matter. Following this, Sec. III reviews the Booster Neutrino Beamline (BNB), where the neutrino flux (in beam-dump mode) is given, and the "time-of-flight" measurement is discussed. In Sec. IV the MiniBooNE detector and simulations are reviewed. Section V we present the event distributions, and describe backgrounds, systematics, and the fit methodology. Finally, the dark matter results are presented in Sec. VI, and a discussion of the implications for both the dark matter and neutrino oscillation searches is given in Sec. VII.

\section{THEORY OF SUB-GeV DARK MATTER}

Light dark matter $\chi$ with a mass below $1 \mathrm{GeV} c^{-2}$ and coupled to ordinary matter through a light mediator particle is a viable and theoretically well-motivated possibility. While it is possible that $\chi$ exists at this scale in isolation, on general grounds one may expect a larger "dark sector" of states. One or more of these additional states may mediate interactions to the Standard Model (SM) and may also play a role in the cosmological production of dark matter, allowing for the correct relic abundance through the standard thermal freeze-out mechanism.

The simplest dark sector scenario of this type is known as vector portal dark matter, in which the interactions of $\chi$ are mediated by a new dark $U(1)$ gauge boson $V_{\mu}$ that kinetically mixes with the ordinary photon [24-29]. In such a model, there are four parameters that govern the properties of dark matter: the dark matter mass $m_{\chi}$, the dark photon mass $m_{V}$, the kinetic mixing angle $\epsilon$, and the dark gauge coupling $g_{D}$. Equation (1) gives the Lagrangian $\mathcal{L}_{V}$ that is added to the SM Lagrangian:

$$
\mathcal{L}_{V}=\mathcal{L}_{\chi}-\frac{1}{4} V_{\mu \nu} V^{\mu \nu}+\frac{1}{2} m_{V}^{2} V_{\mu} V^{\mu}-\frac{\epsilon}{2} F_{\mu \nu} V^{\mu \nu},
$$




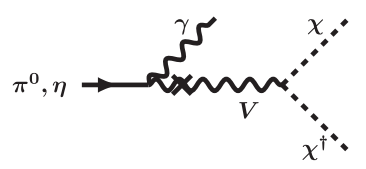

(a) Meson Decay

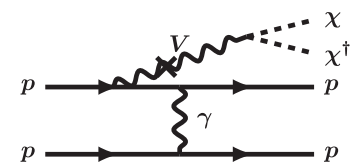

(b) Proton Bremsstrahlung + Vector-Mixing
FIG. 2. Feynman diagrams for the production channels relevant for the MiniBooNE dark matter search [20].

where

$$
\mathcal{L}_{\chi}= \begin{cases}i \bar{\chi} \not \chi-m_{\chi} \bar{\chi} \chi & \text { Dirac fermion } \\ \left|D_{\mu} \chi\right|^{2}-m_{\chi}^{2}|\chi|^{2} & \text { Complex scalar }\end{cases}
$$

and $D_{\mu}=\partial_{\mu}-i g_{D} V_{\mu}$ with the dark matter charge equal to one. The interactions above lead to efficient dark matter annihilation to light SM particles such that the observed dark matter abundance can be explained for certain values of the model parameters. Furthermore, if the dark matter is a complex scalar the annihilation occurs in the $p$-wave and is velocity suppressed [2], evading otherwise strong constraints from the cosmic microwave background [30]. For this reason, the dark matter particle is assumed to be a complex scalar in this work.

The BNB is able to produce dark matter through several mechanisms, illustrated in Fig. 2. They are (i) decay of secondary $\pi^{0}$ or $\eta$ mesons, and (ii) proton bremsstrahlung plus vector-meson mixing. Note that in all cases, the production rate scales as $\epsilon^{2}$ provided $V$ can decay to two on-shell $\chi$. On-shell decay is defined by $m_{V}>2 m_{\chi}$, and is known as the invisible decay mode.

Once the dark matter is produced by one of these mechanisms, it can scatter with nucleons or electrons through a neutral-current channel in the detector via $V_{\mu}$ boson exchange, as depicted in Fig. 3. The scattering rate scales as $\epsilon^{2} \alpha_{D}$, where $\alpha_{D}=g_{D}^{2} / 4 \pi$. The acceleratorproduced dark matter event rate in MiniBooNE scales as $\epsilon^{4} \alpha_{D}$ for on-shell decays in this model.

Another potential dark sector scenario amenable to the MiniBooNE search is leptophobic dark matter $[8,10,11,31,32]$, in which the mediator $V$ couples dominantly to quarks and not leptons. For illustration, a

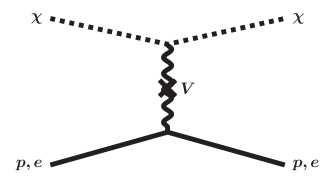

(a) Free Protons or Electrons

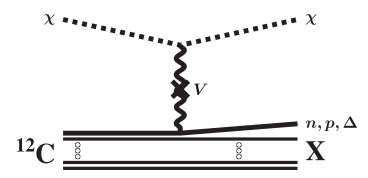

(b) Bound Nucleons
FIG. 3. Feynman diagrams for the dark matter interactions with nucleons and electrons in MiniBooNE. The $\Delta$, in the boundnucleon case, would be observed by its decay products: a pion and a nucleon. simplified scenario is presented in which a vector mediator couples to the baryon number current, with the Lagrangian given in Eq. (2):

$$
\mathcal{L}_{B}=\mathcal{L}_{V}+g_{B} V_{\mu} J_{B}^{\mu}+\cdots,
$$

where

$$
J_{\mu}^{B}=\frac{1}{3} \sum_{i} \bar{q}_{i} \gamma_{\mu} q_{i}
$$

is the sum over all quark species, and $\mathcal{L}_{V}$ [Eq. (1)] is dependent on the baryon gauge coupling $g_{B}\left(g_{D}\right.$ is replaced by $g_{B}$ ). The limit $\epsilon e \ll g_{B}$ gives the leptophobic dark matter scenario. Three parameters will be considered in the interpretation of the presented results: the dark matter mass $m_{\chi}$, the leptophobic vector mediator mass $m_{V}$, and the coupling $\alpha_{B}=g_{B}^{2} / 4 \pi$. Consideration of the dark matter production and scattering rates leads to the conclusion that the event rates scale as $\alpha_{B}^{3}$ for on-shell decays.

It turns out to be challenging to construct a phenomenologically viable UV completion of the leptophobic model with large mediator couplings to the SM. Among other challenges, significant constraints arise as a consequence of the anomalous nature of the vector mediator in the case at hand [33-35], which will provide stronger constraints than the MiniBooNE dark matter search in most UV completions of the model. Nevertheless, the MiniBooNE limits presented here are likely to be of value in certain leptophobic scenarios, e.g., those involving leptophobic scalar mediators.

As we are discussing new light degrees of freedom (d.o.f.) at the (sub-) GeV scale, a variety of constraints from past experiments must be considered. The strongest constraints on the scenarios discussed above arise from fixedtarget/beam-dump experiments, medium-energy $e^{+} e^{-}$ colliders, and meson decays. These constraints were described in detail in Refs. [9,29,36-38] for the vector portal model, and in Refs. [8,34,35] for the leptophobic model.

\section{BOOSTER NEUTRINO BEAMLINE}

The Fermilab Booster delivers $8 \mathrm{GeV}$ (kinetic energy) protons to the BNB target hall. As shown in Fig. 1, when running in on-target mode a secondary beam of mesons is produced that travel through the air-filled decay pipe and decay in flight to produce neutrinos which then travel and interact in the MiniBooNE detector. The intensity of the proton beam can range from $1 \times 10^{12}$ protons per pulse (ppp) to $5 \times 10^{12} \mathrm{ppp}$.

Each pulse has a $53 \mathrm{MHz}$ microstructure that is composed of 82 bunches, and each bunch has a full width at half maximum of $2 \mathrm{~ns}$. Figure 4 overlays an example trace of the BNB pulse microstructure, with an arbitrary offset with neutrino mode $\nu_{\mu}$ charged-current quasielastic (CCQE) interactions in the MiniBooNE detector; see Sec. V for a 


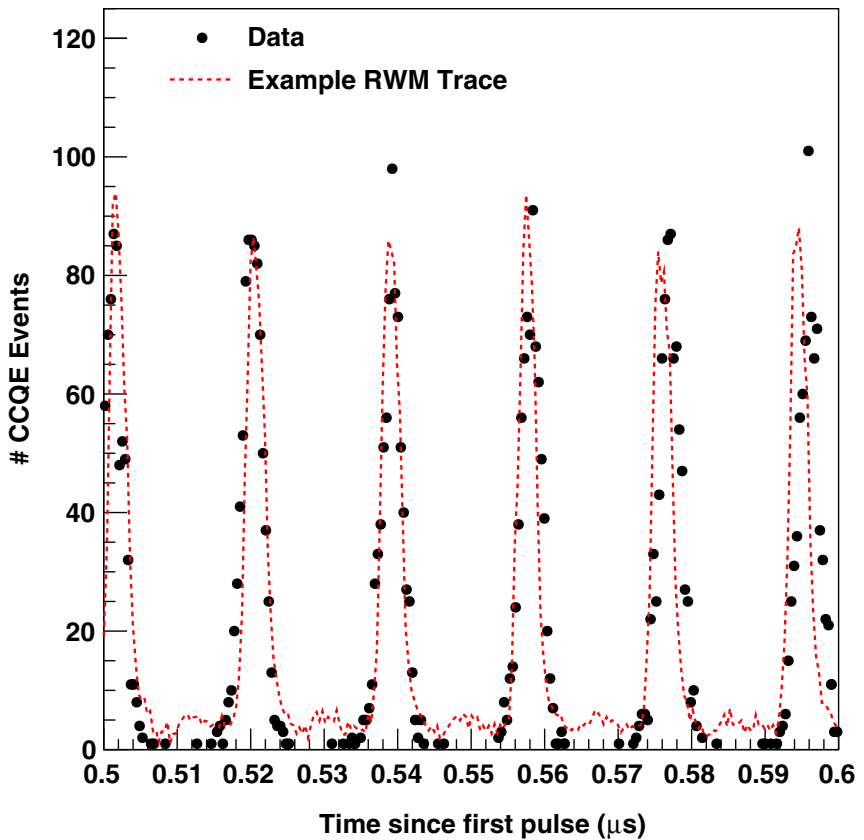

FIG. 4. Zoomed-in example of the BNB pulse microstructure as measured by the RWM. The data points come from neutrinomode $\nu_{\mu}$ charged-current interactions in the MiniBooNE detector during 2015-2016. The example RWM trace is plotted by the readout value of the trace.

definition. The trace and the CCQE data shapes are in good agreement.

Neutrinos are a background for the dark matter search. To reduce the neutrino production coming from the BNB, the primary proton beam was steered above the beryllium target, and into a cooling air gap (which is inside the neck of the aluminum horn). After leaving the horn the protons enter the air-filled decay pipe, and finally reach the beam dump located $50 \mathrm{~m}$ downstream of the target location, as illustrated in Fig. 5. Running in this mode reduces the number of charged mesons that are generated in the thin beryllium target.

The charged mesons that are produced in a thin target will escape and produce decay-in-flight neutrinos, while within the beam dump, the charged mesons are absorbed or decay at rest within a few radiation lengths, as illustrated in Fig. 6. This is in comparison with neutral mesons that will decay in flight due to their short lifetimes. The neutral mesons could decay into a dark photon which would then

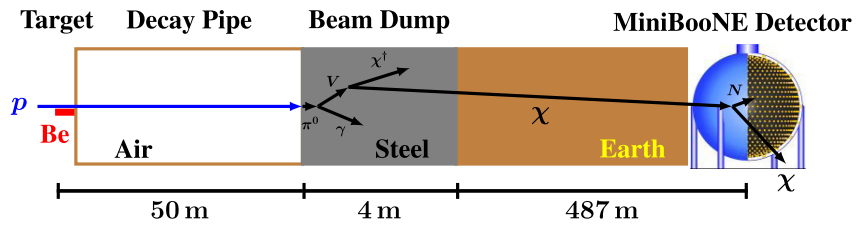

FIG. 5. The production of dark matter in off-target running [20].

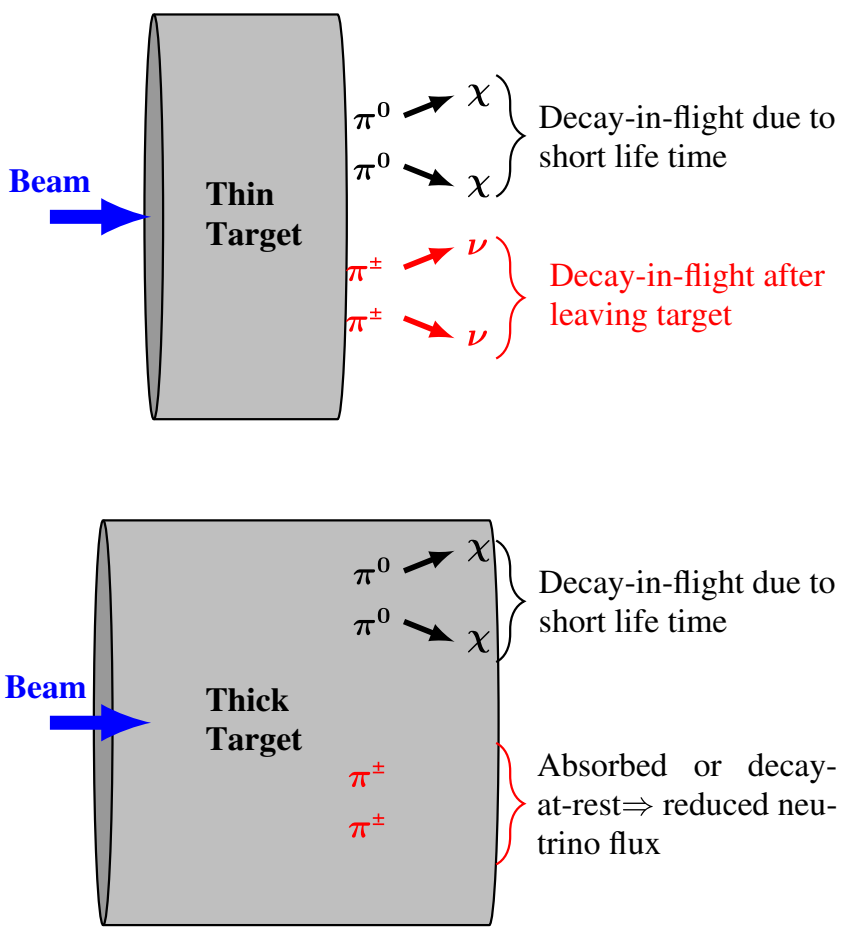

FIG. 6. (Top) Production of dark matter and neutrinos when the beam hits a thin target. (Bottom) The production of dark matter and suppression of neutrino generation when the beam hits a thick target.

decay into two dark matter particles, as shown schematically in Fig. 5. The horn was turned off during this run so no charged particles generated would be (de)focused. For the rest of this paper, this mode of running will be denoted as off-target, since the beryllium target and horn were not removed from the beam line.

The decay pipe and beam dump are buried in crushed aggregate. There is a metal end cap at the downstream end of the decay pipe which prevents aggregate from entering the pipe. The beam dump consists of 104 inches of steel followed by 36 inches of concrete and another 26 inches of steel in the beam direction. A detailed study of the neutrino flux coming from the BNB in on-target mode seen in the MiniBooNE detector using the GEANT4 [39] simulation package BooNEG4BEAM can be found in Ref. [40]. Ontarget running consisted of neutrino, and antineutrino modes. The simulations were updated to study the offtarget beam configuration and are described below.

\section{A. Beam off-target BNB simulation}

BooNEG4BEAM was updated to include materials in the beam line that would have changed the neutrino-mode flux $\Phi_{\nu}$ by less than a percent but are important for the off-target beam configuration. Figure 7 shows a schematic of the beam-line geometry around the target, pointing out the materials that were added. An aluminum window at the end of the horn and a steel end cap with a small gap of air 
1. *Vacuum Pipe

2. *MWTGT Al Foils and Wires

3. ${ }^{\star T i}$ Window

4. *Al Target Back Plate

5. *Be Window

6. *Al Target Base Block

7. *Be Upstream Fin Locator

8. ${ }^{A} \mathrm{Al}$ Bellows Contact Assembly

9. Al Horn

10. Be Target, Fins and Outer Tube

11. *Be Downstream Fin Locator
(9)

(3)

(1)

(2)

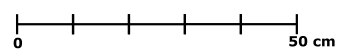

(11)

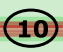

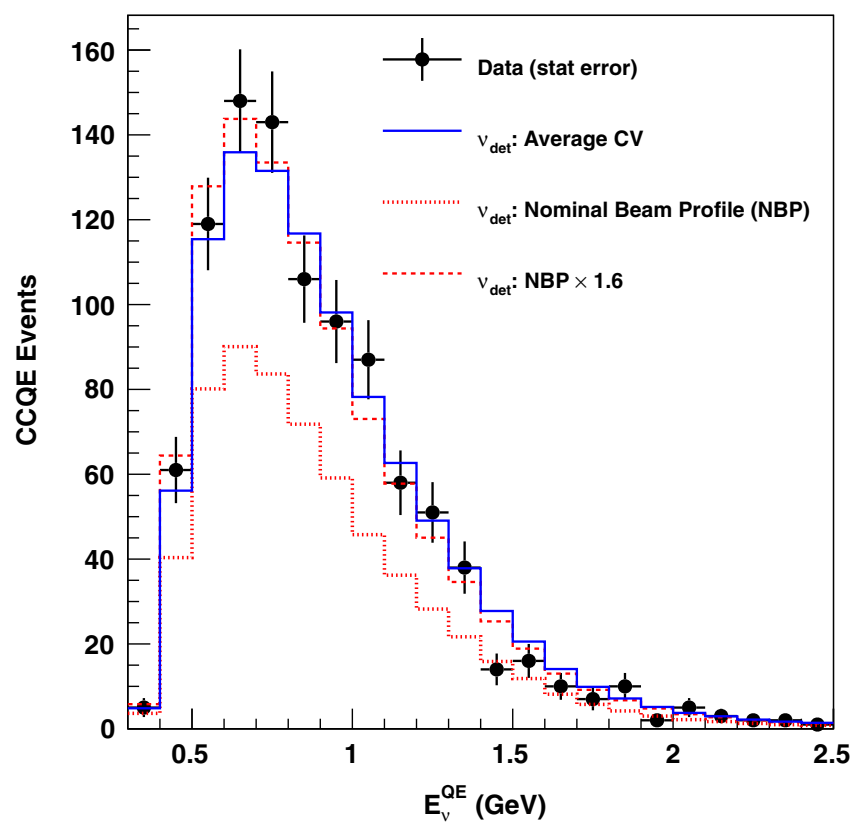

FIG. 8. Comparing CCQE data in off-target mode to three different Monte Carlo predictions for neutrinos interacting in the detector $\left(\nu_{\text {det }}\right)$. The dotted line is the output of the nominal offtarget beam profile, the dashed line is the nominal profile scale by 1.6, and the solid line is the average of the scrapings (Average $\mathrm{CV}$ ) used as the final $\Phi_{\mathrm{Off}}[41] . E_{\nu}^{\mathrm{QE}}$ is defined by Eq. (5).

of the beam to scrape the beryllium target downstream of the $90^{\circ}$ beam-loss monitor. The same study showed, with the low-statistics off-target data, that no distinction could be made between the different scrapings. An average of four potential scraping scenarios produced the needed increase in the number of CCQE events. The average is defined as $\Phi_{\text {Off }}$, as shown in Fig. 10.

Uncertainty in $\Phi_{\text {Off }}$ was determined by $1 \sigma$ excursions around the nominal beam profile scaled by 1.6 so the

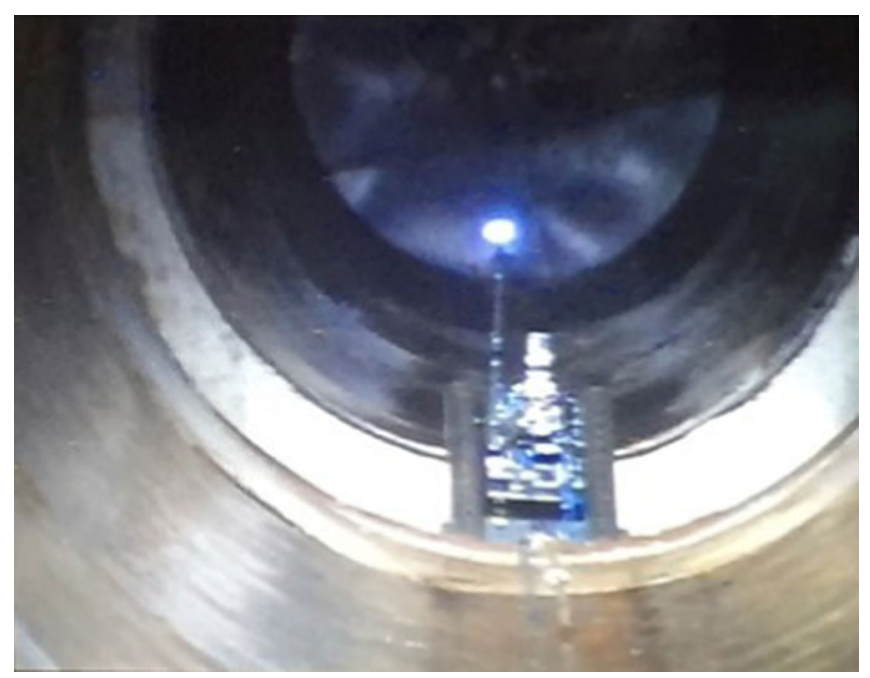

FIG. 9. Picture of FRED at the 25-m absorber. 


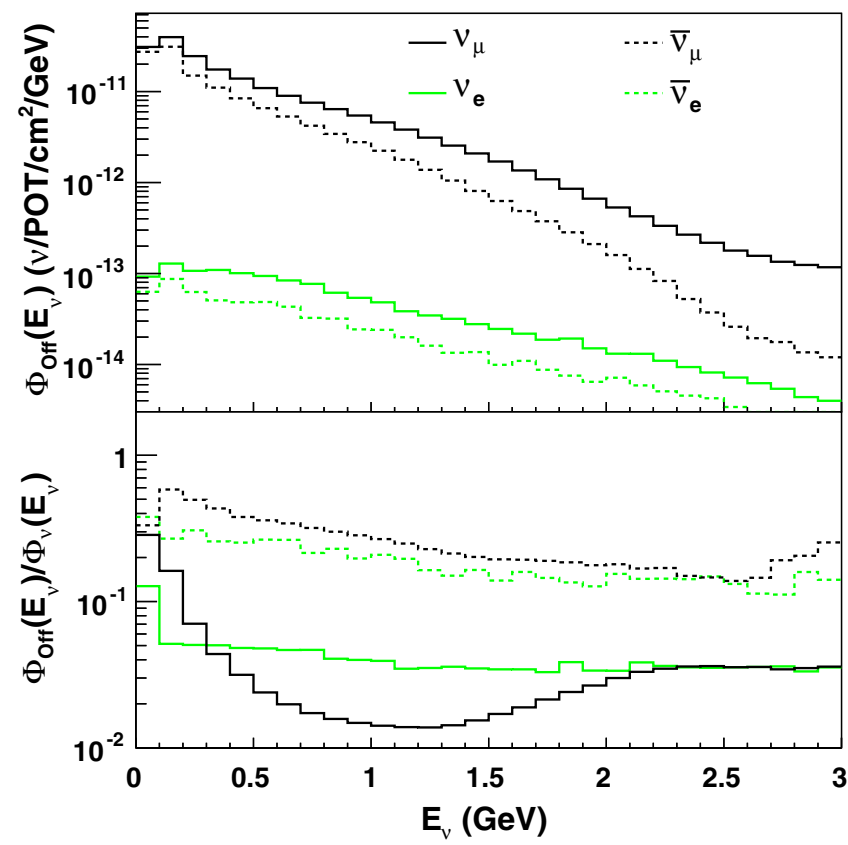

FIG. 10. (Top) The off-target neutrino flux seen by the MiniBooNE detector. (Bottom) The off-target/neutrino flux ratio [20].

central value number of CCQE events matches data, and the four potential scraping scenarios that were averaged to generate $\Phi_{\text {Off }}$. The integrated $\Phi_{\text {Off }}$ with a neutrino energy $E_{\nu}$ between 0.2 and $3 \mathrm{GeV}$ is $(1.9 \pm 1.1) \times$ $10^{-11} \mathrm{\nu} / \mathrm{POT} / \mathrm{cm}^{2}$ with a mean energy of $660 \mathrm{MeV}$. The large uncertainty on $\Phi_{\text {Off }}$ comes from not knowing which scraping scenario is physically happening. Comparing this to the integrated $\Phi_{\nu}$ of $5.0 \times 10^{-10} \nu / \mathrm{POT} / \mathrm{cm}^{2}$ with a mean energy of $830 \mathrm{MeV}$ gives a flux reduction factor of 27. The reduction factor as a function of $E_{\nu}$ and species is shown in Fig. 10. The combination of the flux reduction and the softer spectrum, which has lower neutrino cross sections in the detector, results in an event-rate reduction by a factor of 48 in both CCQE and NCE interactions (cuts given in Table III).

The breakdown of the integrated $\Phi_{\text {Off }}$ for the different neutrino species is given in Table I. While $\Phi_{\nu}$ is made up of $93.6 \% \nu_{\mu}, 5.9 \% \bar{\nu}_{\mu}$, and $0.5 \% \nu_{e}, \bar{\nu}_{e}[40], \Phi_{\text {Off }}$ is composed of $63.7 \% \nu_{\mu}, 35.4 \% \bar{\nu}_{\mu}$, and $0.9 \% \nu_{e}, \bar{\nu}_{e}$. The breakdown of

TABLE I. Beam off-target profile systematic percent error independent of energy for the various neutrino types, including correlations. $\Phi_{\text {Off }}$ integrated over $0.2<E_{\nu}<3 \mathrm{GeV}$.

\begin{tabular}{lrr}
\hline \hline Neutrino species & $\Phi_{\text {Off }}\left(\nu / \mathrm{POT} / \mathrm{cm}^{2}\right)$ & $\%$ of total \\
\hline Total & $(1.9 \pm 1.1) \times 10^{-11}$ & \\
$\nu_{\mu}$ & $(1.2 \pm 0.6) \times 10^{-11}$ & 63.7 \\
$\bar{\nu}_{\mu}$ & $(6.6 \pm 4.7) \times 10^{-12}$ & 35.4 \\
$\nu_{e}$ & $(1.1 \pm 0.9) \times 10^{-13}$ & 0.6 \\
$\bar{\nu}_{e}$ & $(5 \pm 4) \times 10^{-14}$ & 0.3 \\
\hline \hline
\end{tabular}

$\Phi_{\text {Off }}$ by the source material that the secondary beam (Fig. 1) was generated in is $55 \%$ air, $30 \%$ beryllium, $10 \%$ steel, 3\% aluminum, and 2\% concrete. Air and beryllium provide approximately equal contributions to $\Phi_{\text {Off }}$ for $E_{\nu}$ above $500 \mathrm{MeV}$ with almost no contributions from the other materials.

The sensitivity to dark matter depends on the number and distribution of $\pi^{0}$ 's generated in the beam line. Table II gives the total number of $\pi^{ \pm}$per POT as well as the breakdown by material in the beam line for both off-target and neutrino running simulated by BooNEG4BEAM. The simulated $\pi^{0}$ distribution was chosen as the average of the $\pi^{+}$and $\pi^{-}$distributions which has been shown to be in good agreement with actual $\pi^{0}$ distributions [42-44]. Neutrino-mode charged pions are generated evenly in beryllium, steel, and concrete. The concrete surrounds the decay pipe and the steel is primarily located in the beam dump. The charged pions generated in the concrete and steel, if able to decay, will produce low-energy neutrinos and therefore do not contribute much to the on-target neutrino event rate. Off-target charged pions are predominantly produced in steel, which is consistent with the reduction of the neutrino flux. The different scraping scenarios that generate the off-target central value flux changes the number of pions produced in the steel beam dump by less than a percent.

Taking the average of the charged-pion distributions to generate the $\pi^{0}$ distribution, the off-target $\pi^{0}$ distribution will generate a greater dark matter flux than on-target

TABLE II. The breakdown of the number of charged pions per POT and by material in the beam line. A pion was counted if it had a total kinetic energy greater than $1 \mathrm{MeV}$, was traveling in the forward direction, and had a transverse momentum less than $1 \mathrm{GeV} c^{-1}$. Off-target in this table refers to the nominal beam configuration measured by the multiwires, not the average of the four possible scraping scenarios that is used as the off-target neutrino flux.

\begin{tabular}{lrr}
\hline \hline & $\pi^{+}$ & $\pi^{-}$ \\
\hline Off-Target meson/POT & 2.48 & 2.36 \\
Composition & & \\
Air & $3.6 \%$ & $3.0 \%$ \\
Aluminum & $0.2 \%$ & $0.2 \%$ \\
Beryllium & $0.2 \%$ & $0.2 \%$ \\
Concrete & $3.6 \%$ & $4.1 \%$ \\
Dolomite & $0.1 \%$ & $0.1 \%$ \\
Steel & $92.3 \%$ & $92.4 \%$ \\
Neutrino-Mode meson/POT & 2.54 & 2.51 \\
Composition & & \\
Air & $1.7 \%$ & $1.4 \%$ \\
Aluminum & $5.3 \%$ & $5.2 \%$ \\
Beryllium & $29.5 \%$ & $27.6 \%$ \\
Concrete & $28.0 \%$ & $27.6 \%$ \\
Dolomite & $0.1 \%$ & $0.2 \%$ \\
Steel & $35.4 \%$ & $38.0 \%$ \\
\hline \hline
\end{tabular}




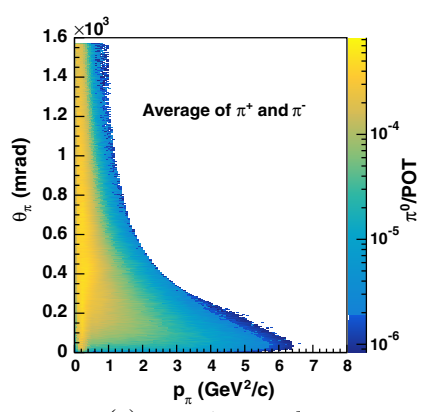

(a) neutrino-mode

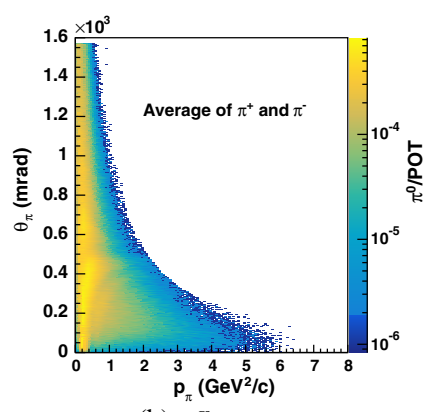

(b) off-target

FIG. 11. The $\theta_{\pi^{0}}$ vs $p_{\pi^{0}}$ distributions from BooNEG4BEAM used for generating dark matter candidate events. The color scale gives the number of pions per delivered POT in each bin.

because more of the pions are generated at the beam dump transversely closer to the center of the beam spot. Figure 11 shows the angle vs total pion momentum for the $\pi^{0}$ distribution used as input to the dark matter simulations, discussed in Sec. VI. The total integral is dominated by low-momentum pions, where pions were simulated down to a total kinetic energy of $1 \mathrm{MeV}$.

For the $\eta$-meson distribution the $\pi^{0}$ distribution was reweighted by setting the total momentum of the $\eta$ meson to be

$$
p_{\eta}=\sqrt{E_{\pi^{0}}^{2}-m_{\eta}^{2}}
$$

where $E_{\pi^{0}}$ is the total energy of the $\pi^{0}$ being reweighted, and $m_{\eta}$ is the mass of the $\eta$ meson. Only $\pi^{0}$ events that satisfy $E_{\pi^{0}}>m_{\eta}$ were used in the reweighting scheme. The momentum vector $\mathbf{p}$ for the $\eta$ meson is then calculated by

$$
\mathbf{p}_{\eta}=\mathbf{p}_{\pi^{0}} \frac{p_{\eta}}{p_{\pi^{0}}} .
$$

A systematic test was preformed to generate the $\eta$-meson distribution by doing the same procedure above but starting with the predicted off-target kaon distribution instead of the $\pi$ distribution. An independent simulation using PYTHIA [45] predicted the $\eta$ distribution to closely match the distribution obtained by reweighting of the kaon distribution. The final confidence level limits, discussed in Sec. VI, showed no change in the predicted sensitivity for a slice of the dark matter parameter space. The predicted $\eta$ distribution used for the final analysis was the one that used the $\pi$ distribution, because there are smaller uncertainties on the $\pi$ production.

A particle list of $\pi^{0}$ and $\eta$ mesons with their 4-momentum and 4-position information was passed to the dark matter simulation (see Sec. VI) as input for neutral meson production of dark matter.

\section{B. Bunch time}

As the beam travels down the beam line the protons induce an image charge on the vacuum pipe. A resistive

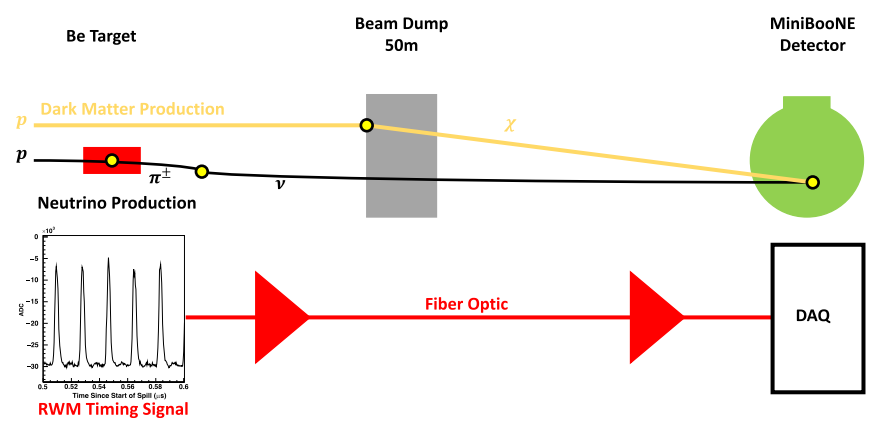

FIG. 12. Illustration showing how the RWM time signature is passed to the detector. Production of neutrinos and dark matter particles are also shown for comparison. Heavy dark matter will arrive later than the neutrinos.

wall current monitor (RWM) right in front of the beryllium target uses the image charge to measure the longitudinal bunch shape and the time the bunch hits the target $[46,47]$. The RWM design is based on the RWMs that were installed in the Fermilab Main Injector. The intensity of the individual $2 \mathrm{~ns}$ bunches are measured to $1 \%$ and the timing is known to less than $1 \mathrm{~ns}$. The RWM signals, one for each bunch, are saved for each data acquisition (DAQ) window (described in the next section).

The RWM signal is sent to the MiniBooNE DAQ by an optical fiber, as shown schematically in Fig. 12. For each reconstructed event a time is calculated to the first RWM bunch that passes threshold, as shown in Fig. 4. The bunch time is the remainder of the time of the event subtracted by the time of the RWM divided by 18.9 ns; see Fig. 13 .

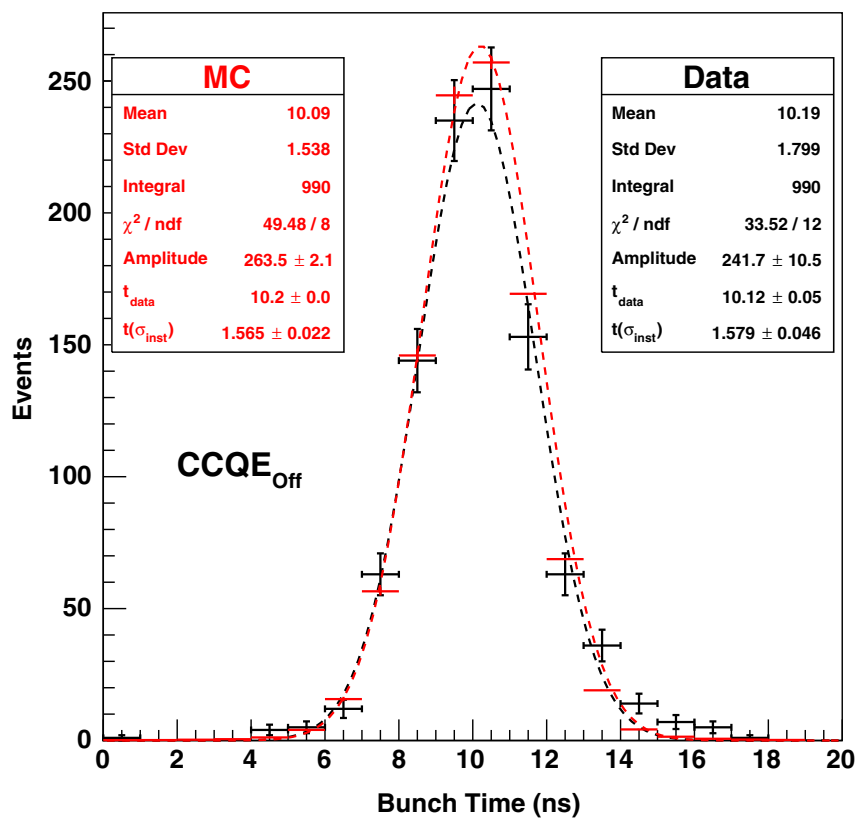

FIG. 13. Comparison of simulated and measured CCQE bunch times after applying $\delta t\left(\sigma_{\text {inst }}\right)$ and $\delta t_{\text {data }}$ calibrations (see text). Only statistical errors are shown. 


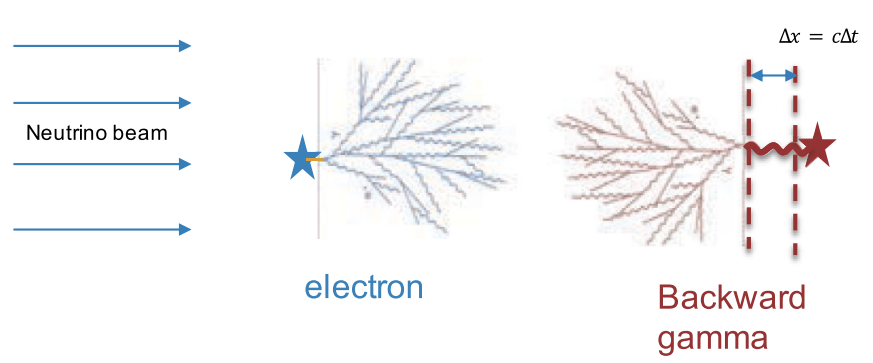

FIG. 14. Illustration of the timing difference between an electron event and a backward-going photon.

The measured bunch time is a time-of-flight measurement, where two regions are defined: in-time and out-oftime. The in-time region is between 5.6 and $14.5 \mathrm{~ns}$ determined from the off-target CCQE data mean and standard deviation.

Cherenkov light has a timing resolution of $\sim 1.5 \mathrm{~ns}$, while the timing resolution of scintillation light is $\sim 4.2 \mathrm{~ns}$ from the lifetime of the scintillation light. The bunch times of photon events has the same timing resolution as that of muon and electron events but are shifted later in time because the photons travel some distance before converting into an electromagnetic shower in the detector, as illustrated in Fig. 14.

The beam-unrelated backgrounds and beam-related events that happen outside the detector (dirt) have flat distributions in bunch time, as shown in Fig. 15. This allows for an analysis cut to remove more background events or to look for a bump in the out-of-time region for new physics. The timing information could also be used as

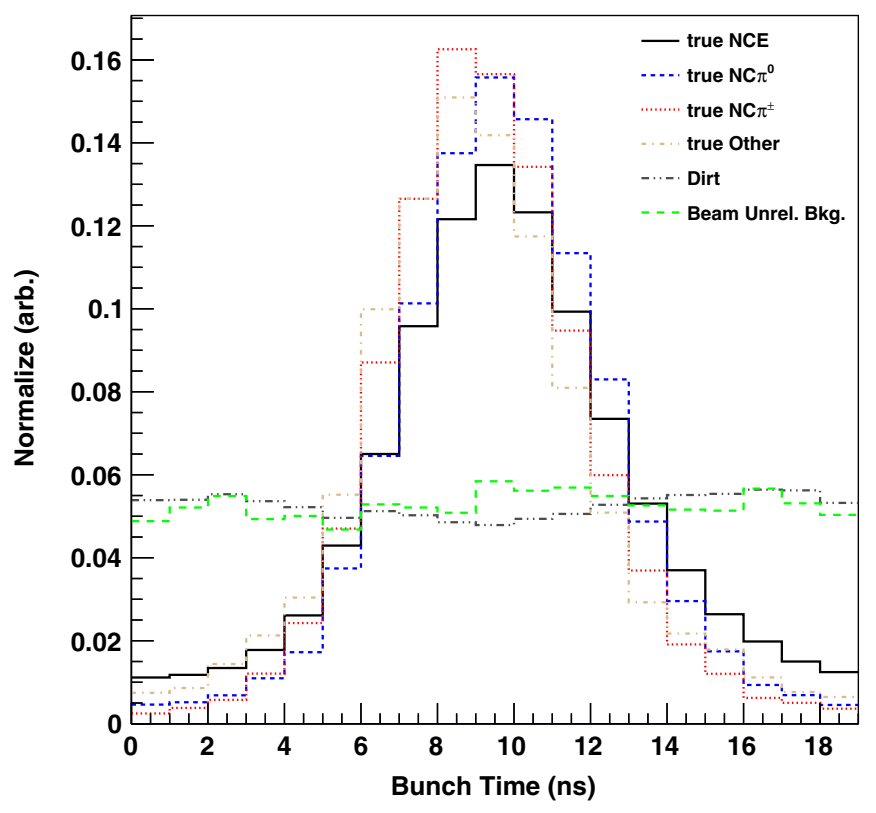

FIG. 15. Comparison of the bunch-time shape for different event types, that pass NCE selection cuts, as predicted by the detector simulation. an extra particle identification parameter, because different event types, or even final-state particles, have different shapes in bunch time for the same selection cuts, as shown in Fig. 15.

\section{Simulating bunch time}

The simulated bunch time $T_{\text {bunch }}$ was calculated by,

$T_{\text {bunch }}=\delta t_{\text {reco }}-\delta t_{Z}+\delta t_{\text {dcy }}-\delta t\left(\sigma_{\mathrm{RF}}\right)-\delta t\left(\sigma_{\text {inst }}\right)-\delta t_{\text {data }}$,

where $\delta t_{\text {reco }}$ is the difference between true and reconstructed time, $\delta t_{Z}$ is the time it takes light to travel from the $z=0$ plane to the plane the event occurred in, and $\delta t_{\mathrm{dcy}}$ is the difference between the time it takes light to get from the target to where the neutrino occurred and the simulated decay chain time. $\delta t\left(\sigma_{\mathrm{RF}}\right)$ is a number based on the time jitter of the radio-frequency bunch structure, measured to be $1.15 \mathrm{~ns} . \delta t\left(\sigma_{\text {inst }}\right)$ is the jitter from MiniBooNE instrumentation and $\delta t_{\text {data }}$ is the mean bunch-time difference between simulation and data. Both $\delta t\left(\sigma_{\text {inst }}\right)$ and $\delta t_{\text {data }}$ were tuned to off-target CCQE data, as shown in Fig. 13.

If dark matter has a mass approximately greater than $25 \mathrm{MeV}^{-2}$, it could reach the detector in the out-of-time region. This would distort the bunch time distribution. The bunch time is used in this analysis as an extra constraint on the possible dark matter parameters.

\section{MINIBOONE DETECTOR}

The MiniBooNE detector, described in Ref. [48], is a Cherenkov and scintillation tracking detector designed to search for $\nu_{e}$ and $\bar{\nu}_{e}$ appearance oscillations at short baseline [21]. It is located $541 \mathrm{~m}$ downstream from the center of the BNB neutrino target. As described above, the majority of dark matter production is expected to occur at the 50-m absorber whose front face is $491 \mathrm{~m}$ from the center of the detector. The proton beam is aligned $1.9 \mathrm{~m}$ below the center of the detector during normal neutrino running.

The detector is a $12.2 \mathrm{~m}$ diameter spherical tank filled with 818 tons of Marcol 7 light mineral oil $\left(\mathrm{C}_{n} \mathrm{H}_{2 n+2}\right.$ where $n \approx 20$ ). No additives were introduced in the mineral oil, but there remain small levels of fluorescent contaminants. There is a spherical optical separation with a radius of $5.476 \mathrm{~m}$ centered within the main volume. The outer "veto" region contains the same mineral oil as the inner "tank" region despite being optically separated.

The index of refraction of the oil was measured to be 1.47, yielding a Cherenkov light threshold for particles with $\beta>0.68$. For protons (electrons) this is approximately $280 \mathrm{MeV}(150 \mathrm{keV})$. The impurity fluors contribute enough scintillation light to push the proton detection threshold well below this.

The inner region is viewed by 1280 inward-facing 8-inch photomultiplier tubes (PMTs). These PMTs are mounted 
on the inner surface of the optical barrier and provide $11.3 \%$ photocathode coverage. The outer region is viewed by 240 PMTs arranged in pairs around the outside of the optical barrier. These outer-region PMTs are of the same type as the inner region PMTs.

The light signal read out by the PMTs is sent to custombuilt electronics where the signal is amplified, discriminated, and then digitized. The electronics ("QT" boards [49]) both integrate the total charge and extract the start time of the digitized pulse. Threshold was equivalent to about 0.1 photoelectrons. All the hits from all the PMTs are accumulated into buffers to await a trigger decision from the logic. The multiplicity of PMT hits and external signals are used to create various triggers for physics and calibrations.

When a trigger occurs, $19.2 \mu$ s of PMT hits are extracted from the QT boards. The physics trigger was a Fermilab accelerator signal that signals when protons are being delivered to the BNB area. The $1.6 \mu \mathrm{s}$ beam spill is placed $5 \mu \mathrm{s}$ after the start of data acquisition. Therefore, the intrinsic cosmic-ray background activity is adequately measured. The remaining $12 \mu \mathrm{s}$ of time measures the neutrino-induced muon decays which have a lifetime of $2.2 \mu \mathrm{s}$.

\section{A. Detector simulation}

The detector simulation was split up into neutrino interaction and detector response. The neutrino interaction simulation used a modified version of the NUANCE V3 neutrino event generator for simulating neutrino interactions in $\mathrm{CH}_{2}$ [50]. Descriptions of the relevant NUANCE models and uncertainties were given in Refs. [51] and [22] for NCE and CCQE respectively and in Ref. [52] for neutral-current single $\pi^{0}$ production $\left(\mathrm{NC} \pi^{0}\right)$. In summary, the relativistic Fermi gas model of Smith and Moniz is used to describe both NCE and CCQE events, while the Rein and Sehgal models [53,54] are used to predict $\mathrm{NC} \pi^{0}$. Pion absorption and charge exchange are included in generating the final-state particles. The axial form factor is assumed to be of dipole form with an axial mass $M_{a}$ and a Pauliblocking parameter $\kappa$ is introduced as an extra d.o.f. to model low 4-momentum transfer $Q^{2}$ events in MiniBooNE correctly [22].

MiniBooNE used $M_{A}^{\text {eff }}=1.23 \pm 0.20 \mathrm{GeV} c^{-2}$ and $\kappa=$ $1.019 \pm 0.011$ for the simulations generated for neutrinomode publications. In Ref. [22] $M_{A}^{\text {eff }}$ and $\kappa$ were measured to be $1.35 \pm 0.17 \mathrm{GeV} \mathrm{c}^{-2}$ and $1.007 \pm 0.012$, respectively, with an extra 1.08 normalization factor to match simulations with data. For this analysis all detector and dirt simulated events were reweighted to these updated measured values, while only true CCQE events include the normalization factor.

The detector response is modeled with a GEANT3 [55] simulation described in Ref. [48].

\section{Definition of true interactions}

The dark matter simulation (BDNMC) that is used (Sec. VI) does not include a nuclear model or final state interactions. In order to connect the NUANCE and detector simulations to BDNMC, "true" neutrino interactions are defined by the output of a neutrino interaction before any final-state interactions or nuclear model are considered. This makes the definitions used by BDNMC and NUANCE the same.

It should be noted that the weighting scheme to produce a predicted dark matter spectrum coming from the detector simulations is discussed in Sec. VI. The procedure applies the nuclear model and the model for final-state interactions that are in NUANCE to BDNMC to correctly determine the reconstructed dark matter distribution.

\section{EVENT DISTRIBUTIONS}

This analysis consists of four different selection cuts: CCQE, NCE, $\mathrm{NC} \pi^{0}$, and neutral-current neutrino-electron elastic scattering $(\nu-e)$. Because of final-state interactions the events that pass these selection cuts are CCQE-like, $\mathrm{NCE}$-like, $\mathrm{NC} \pi^{0}$-like, and $\nu$-e-like events. For simplicity, for the rest of this paper we will leave off the "-like" when referring to the events that passed the cuts. CCQE candidate events, defined by seeing a primary muon followed by the decay electron, are used to determine the neutrino event rate. $\mathrm{NCE}, \mathrm{NC} \pi^{0}$, and $\nu$-e are considered signal channels. Table III gives selection criteria for each selection cut.

NCE cuts follow from the MiniBooNE antineutrinoNCE analysis [56] with the addition of NCE7, a previous trigger activity cut. A subevent is defined as a group of hits where no hits are separated by more than $10 \mathrm{~ns}$ and the group has no less than 10 hits. Only a single nucleon is expected for NCE interactions, which is why the NCE1 cut allows only one subevent within the $19.2 \mu \mathrm{s}$ DAQ window. NCE2 makes sure that the subevent falls within the beam window inside the DAQ window. NCE3 requires a minimal number of tank hits needed for reconstruction and a maximum number of veto hits for beam-unrelated background rejection. NCE4 sets the fiducial volume, and NCE5 separates scintillation-like events from Cherenkovlike events based on the response time of each process. NCE6 selects the kinetic energy parameter space for the analysis. NCE7 is used to further remove beam-unrelated backgrounds by looking at events that happen in the detector before the beam trigger is turned on. The cut is set to reject all events that have a trigger of greater than or equal to 60 hits in the detector within $10 \mu$ s before the event trigger. The efficiency of NCE7 for beam-related events that passed the previous cuts is $(95.3 \pm 0.2) \%$, while beam-unrelated backgrounds are reduced by $42.5 \%$ [41].

NCE selection cuts are $30 \%$ efficient at detecting true $\mathrm{NCE}, \mathrm{NC} \pi^{0}$ and neutral-current single charged pion $\left(\mathrm{NC} \pi^{ \pm}\right)$events with an energy transfer greater than or 
TABLE III. Selection cuts for the various channels in this analysis.

\begin{tabular}{|c|c|}
\hline Cut \# & Description \\
\hline \multicolumn{2}{|l|}{$C C Q E$} \\
\hline 1 & $\#$ subevents $=2$ \\
\hline 2 & $\begin{array}{l}1 \text { st sub, \# tank }>200 \text { and } \\
\text { all subevents, \# veto hits }<6\end{array}$ \\
\hline 3 & 1 st sub, reconstructed vertex radius $<500 \mathrm{~cm}$ \\
\hline 4 & 1st sub, event time window $4.4<T(\mu$ s $)<6.4$ \\
\hline 5 & 1st sub, $\mu / e$ log-likelihood ratio $>0$ \\
\hline 6 & 1 st sub, kinetic energy $T>200 \mathrm{MeV}$ \\
\hline 7 & $\begin{array}{l}\mu \text {-e vertex distance }>100 \mathrm{~cm} \text { and } \\
>\left(500 T_{\mu}(\mathrm{GeV})-100\right) \mathrm{cm}\end{array}$ \\
\hline \multicolumn{2}{|r|}{ 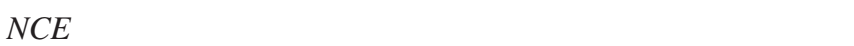 } \\
\hline 1 & $\#$ subevents $=1$ \\
\hline 2 & $\#$ tank hits $>12$ and \# veto hits $<6$ \\
\hline 3 & Reconstructed vertex radius $<500 \mathrm{~cm}$ \\
\hline 4 & event time window $4.4<T(\mu \mathrm{s})<6.4$ \\
\hline 5 & $p / e$ time log-likelihood ratio $<0.42$ \\
\hline 6 & kinetic energy $35<T(\mathrm{MeV})<650$ \\
\hline 7 & $<60$ hits $10 \mu$ s before event trigger \\
\hline \multicolumn{2}{|l|}{$\mathbf{N C} \pi^{0}$} \\
\hline 1 & $\#$ subevents $=1$ \\
\hline 2 & $\#$ tank hits $>200$ and \# veto hits $<6$ \\
\hline 3 & event time window $4<T(\mu \mathrm{s})<7$ \\
\hline 4 & Reconstructed vertex radius $<500 \mathrm{~cm}$ (e fit) \\
\hline 5 & $\mu / e$ log-likelihood ratio $>0.05$ \\
\hline 6 & $e / \pi^{0} \log$-likelihood ratio $<0$ \\
\hline 7 & $80<m_{\gamma \gamma}\left(\mathrm{MeV}^{-2}\right)<200$ \\
\hline \multicolumn{2}{|l|}{$\nu-e$} \\
\hline 1 & $\#$ subevents $=1$ \\
\hline 2 & $\#$ tank hits $>20$ and \# veto hits $\leq 2$ \\
\hline 3 & event time window $4.4<T(\mu \mathrm{s})<6.4$ \\
\hline 4 & Reconstructed vertex radius $<500 \mathrm{~cm}$ \\
\hline 5 & visible energy $75 \leq E_{\mathrm{vis}}^{e}(\mathrm{MeV}) \leq 850$ \\
\hline 6 & reconstructed angle $\cos \theta_{e} \geq 0.9$ \\
\hline 7 & $\mu / e$ log-likelihood ratio: See text \\
\hline 8 & e time log-likelihood $\leq 3.6$ \\
\hline 9 & Scintillation / Cherenkov Ratio $\leq 0.55$ \\
\hline \multirow[t]{2}{*}{10} & Distance to wall $\geq 210 \mathrm{~cm}$ \\
\hline & For events with \# tank hits $>200$ \\
\hline 11 & $e / \pi^{0}$ log-likelihood ratio $>-6.25 \times 10^{-3}$ \\
\hline 12 & $m_{\gamma \gamma} \leq 80 \mathrm{MeV} c^{-2}$ \\
\hline
\end{tabular}

equal to $90 \mathrm{MeV}$ and that interact in the fiducial volume. The effects below $90 \mathrm{MeV}$ were discussed in the previous section. NCE selection cuts result in 95\% pure true NCE, $\mathrm{NC} \pi^{0}$, and $\mathrm{NC} \pi^{ \pm}$events. Table IV gives the breakdown of efficiency for the different true channels, and Fig. 16 shows the efficiency as a function of energy transfer.

True $\nu$ - $e$ events are very forward. $\mathrm{A} \cos \theta_{e}>0.9$ cut was used to have a control region to estimate the background distribution in the signal region, defined by $\cos \theta_{e}>0.99$. The $\nu$-e selection cuts have a stricter number of veto hits $(\nu-e 2)$ than NCE along with a distance to the wall cut
TABLE IV. The efficiency for NCE and $\mathrm{NC} \pi^{0}$ selection cuts for different true signal channels. Only true events that interact in the fiducial volume $r<500 \mathrm{~cm}$ are considered. For true NCE interactions the efficiency given in the parentheses is for energy transfer $>90 \mathrm{MeV}$.

\begin{tabular}{lcl}
\hline \hline True interaction & \multicolumn{2}{c}{ Selection cut efficiency (\%) } \\
\hline & $\mathrm{NCE}$ & $\mathrm{NC} \pi^{0}$ \\
$\mathrm{NCE}$ & $11(37)$ & $<0.1(0.4)$ \\
$\mathrm{NC} \pi^{0}$ & 13 & 15 \\
$\mathrm{NC} \pi^{ \pm}$ & 20 & 3 \\
Total & $12(30)$ & $1(4)$ \\
\hline \hline
\end{tabular}

$(\nu-e 10)$ to remove dirt events. The selections $\nu-e 8$ and $\nu-e 9$ are used to reduce the NCE background. The selection $\nu$-e 7 rejects muon background and uses the same values as that for the oscillation analysis [57,58]. The selections $\nu$-e 11 and $\nu-e 12$ are used to remove high-multiplicity events with a $\pi^{0}$. Events with less than 200 tank hits automatically pass $\nu$-e 11 and $\nu$-e 12 for the cuts are only applied to highmultiplicity events.

The selected $\nu$-e distribution is beam-unrelated background free because of $\nu-e 5$, which sets the minimal reconstructed visible energy $E_{\mathrm{vis}}^{e}$ above the end point of the electron from muon decay. The high- $E_{\mathrm{vis}}^{e}$ cut in $\nu$-e 5 was tuned to maximize the efficiency times purity of the $\nu-e$ sample in the signal region. Lowering the $E_{\mathrm{vis}}^{e}$ will allow more of the predicted dark matter, but the increase in the beam-unrelated backgrounds decreases the sensitivity.
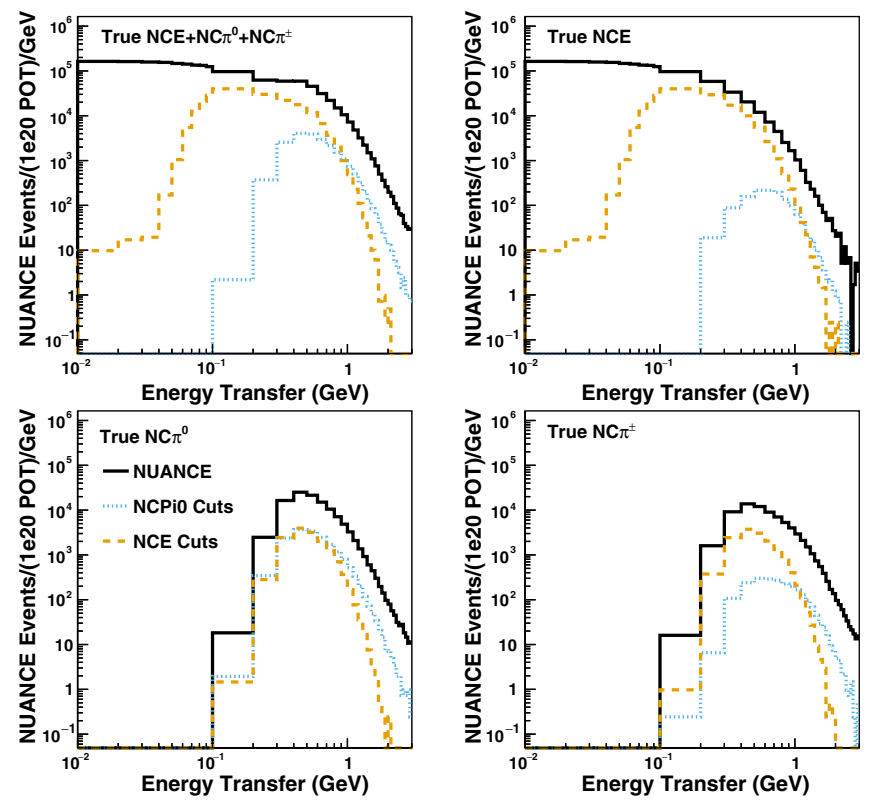

FIG. 16. The expected number of events before and after NCE and $\mathrm{NC} \pi^{0}$ cuts for true $\mathrm{NCE}$, true $\mathrm{NC} \pi^{0}$ and true $\mathrm{NC} \pi^{ \pm}$ interactions. NCE cuts are just as efficient at detecting true $\mathrm{NC} \pi^{0}$ events at low energy transfer because of the pion absorption in the nucleus. 

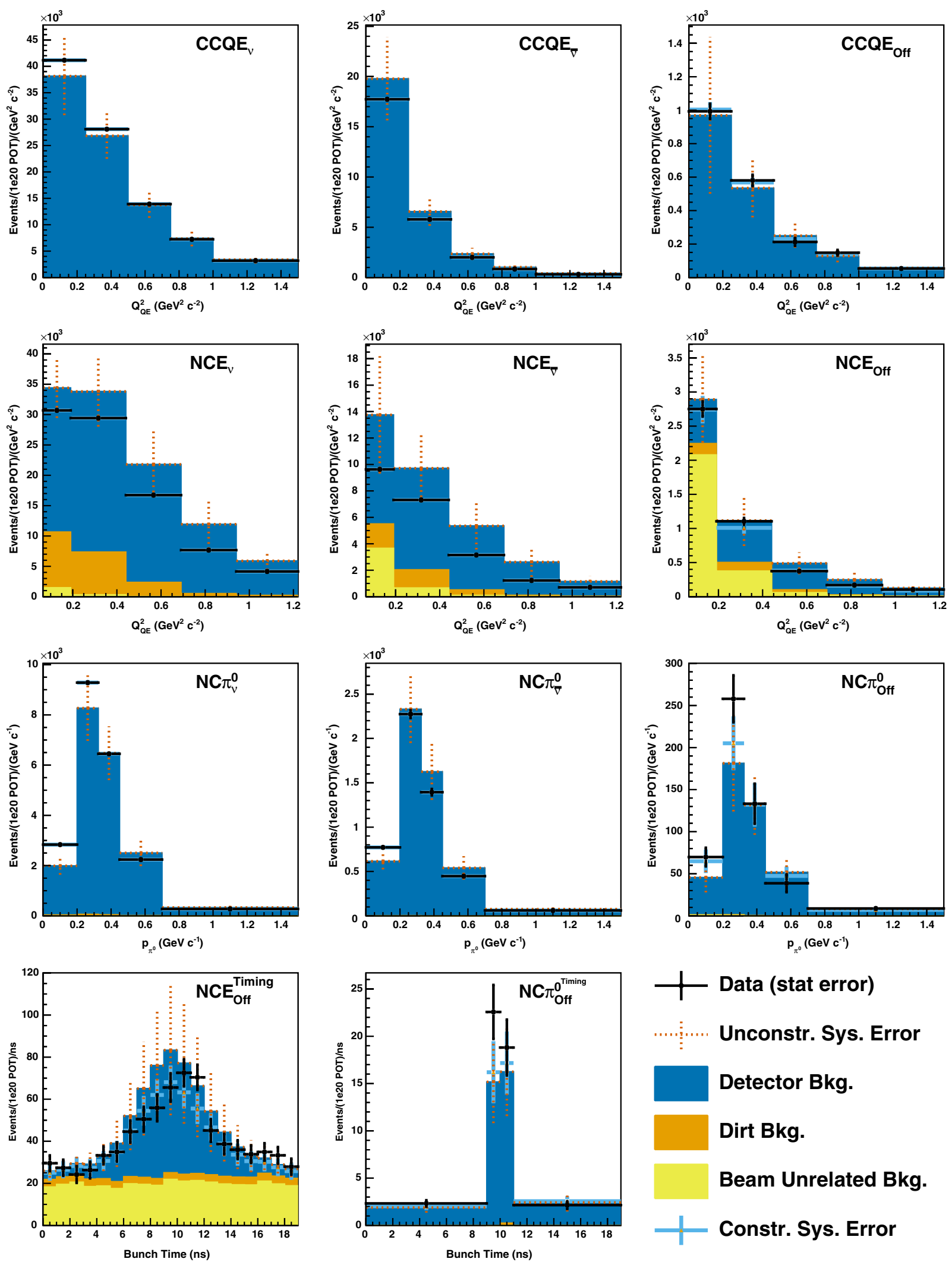

FIG. 17. CCQE, NCE, and $\mathrm{NC} \pi^{0}$ distributions for neutrino, antineutrino and off-target modes. The points are data with statistical errors. The triangles with light lines are constrained predictions with constrained systematic errors. The dotted lines are the total predictions with unconstrained systematic errors. The backgrounds that add up to give the total predictions are given by the stacked histogram. 
The $\nu$-e selection cuts are $15 \%$ efficient and $63 \%$ pure in the signal region for true $\nu-e$ events that interact inside the fiducial volume.

About $40 \%$ of the $\nu$-e candidate events, $\cos \theta_{e}>0.9$, also pass the neutrino oscillation selection cuts as employed in previous analyses [21,57]. The majority of the events that pass $\nu$-e but not oscillation selection cuts come from the lower number of tank hits and $E_{\mathrm{vis}}^{e}$ cuts, along with having no $\pi^{0}$ cuts for events with less than 200 tank hits. Applying the neutrino oscillation selection cuts to the off-target data is discussed in Sec. V C 3 and Sec. VII B.

The cuts for CCQE candidate events are similar to the cuts from Ref. [22] with the addition of the 200-tank-hit cut on the first subevent. The cuts for $\mathrm{NC} \pi^{0}$ candidate events are the same as the cuts from Ref. [52], except for a wider event timing cut. $\mathrm{NC} \pi^{0}$ selection cuts are $4 \%$ efficient and $86 \%$ pure for true $\mathrm{NCE}, \mathrm{NC} \pi^{ \pm}$, and $\mathrm{NC} \pi^{0}$ events. See Table IV and Fig. 16 for the breakdown by true interaction channel and as a function of energy transfer.

The subscripts $\nu, \bar{\nu}$, or "off" will be added to the distribution label when specifically mentioning events after passing cuts from neutrino, antineutrino, or off-target modes respectively.

\section{A. Backgrounds}

There exist two categories of backgrounds: beam-related and beam-unrelated. The beam-unrelated backgrounds are measured by a $2 \mathrm{~Hz}(10-15 \mathrm{~Hz})$ random trigger for ontarget (off-target) running, and scaled by the ratio of the number of beam triggers with POT delivered to the number of random triggers. Knowing that beam-unrelated backgrounds were going to be more significant, the random trigger data-taking rate was increased for off-target running. Beam-related backgrounds can be further split into events that occur in the detector and dirt events (see Sec. III B). For this analysis, all neutrino interactions were considered background.

Beam-unrelated backgrounds were overlaid on top of simulated beam events to correctly simulate the rejection of beam events that have beam-unrelated backgrounds in the same DAQ window. The rate of events passing the one subevent and number of veto hits less than six cuts from the random trigger increased by $3.8 \%$ from neutrino mode to off-target mode. Figure 17 shows the number of events as a function of the dependent variables for CCQE, NCE, and $\mathrm{NC} \pi^{0}$ broken down by predicted background. Also shown are the timing distributions for $\mathrm{NCE}_{\text {Off }}$ and $\mathrm{NC} \pi^{0}$ off. The systematics shown in Fig. 17 are the total systematic uncertainties before constraints are applied (see Sec. V B). Looking at the three NCE distributions the relative percentage of beamunrelated backgrounds increases as the neutrino interaction rate decreases. The resulting $\nu-e$ distributions are shown in Fig. 18.
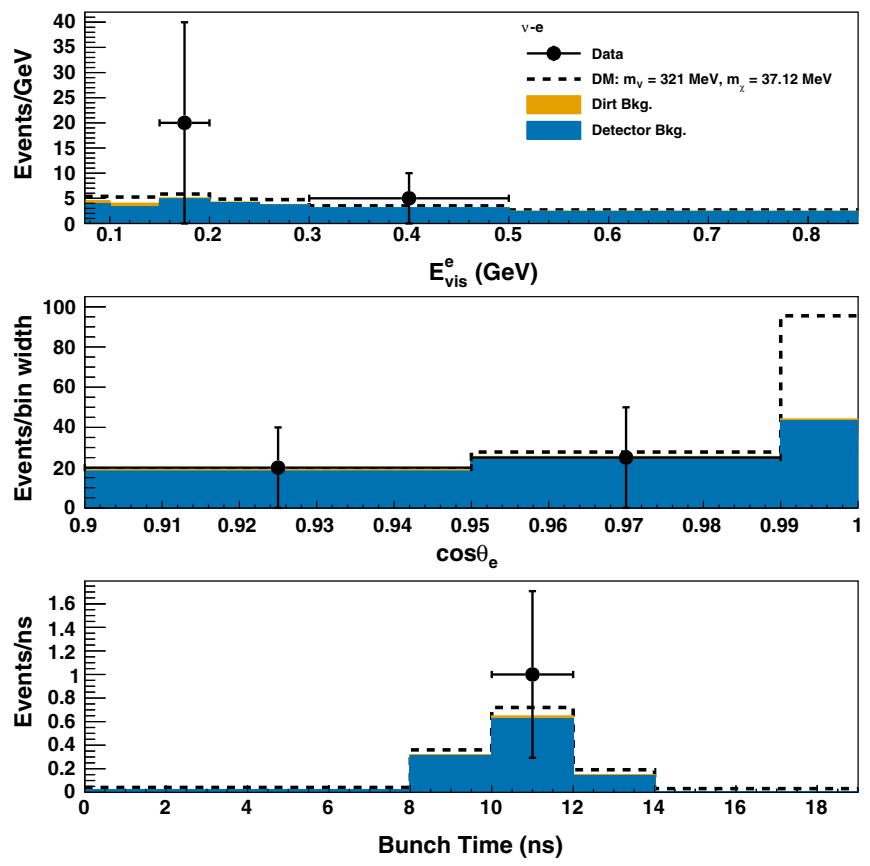

FIG. 18. The (top) visible electron energy $E_{\mathrm{vis}}^{e}$, (middle) electron angle $\cos \theta_{e}$, and (bottom) bunch time distributions that pass $\nu$ - $e$ cuts for off-target mode. The prediction was scaled to match the number of data events for $0.9 \leq \cos \theta_{e}<0.99$. An example dark matter prediction is given (dashed line) to illustrate how forward the resulting electron is expected to be.

\section{B. Systematic uncertainties}

The study of systematic uncertainties considered the correlations between the $\mathrm{NCE}, \mathrm{NC} \pi^{0}$, and CCQE distributions for neutrino, antineutrino, and off-target modes, as well as the timing distributions for $\mathrm{NCE}_{\mathrm{Off}}$ and $\mathrm{NC} \pi^{0}$ Off denoted by $\mathrm{NCE}_{\text {Off }}^{\text {Timing }}$ and $\mathrm{NC}^{0}{ }_{\text {Off }}^{\text {Timing }}$ respectively. Table $\mathrm{V}$

TABLE V. The total unconstrained error broken down by source and distribution. The total constrained error for $\mathrm{NCE}_{\mathrm{Off}}$ is $6.4 \%$ and $11.0 \%$ for $\mathrm{NC} \pi^{0}$ off.

\begin{tabular}{|c|c|c|c|c|c|}
\hline \multicolumn{6}{|c|}{ Source unconstrained total uncertainty $(\%)$} \\
\hline Distribution & $\nu$ flux & $\begin{array}{c}\text { Cross } \\
\text { section }\end{array}$ & $\begin{array}{c}\text { Detector } \\
\text { model }\end{array}$ & $\begin{array}{c}\text { Total } \\
\text { systamatic }\end{array}$ & Statistical \\
\hline \multicolumn{6}{|c|}{ Neutrino Mode } \\
\hline $\mathrm{CCQE}_{\nu}$ & 5.9 & 16.2 & 3.3 & 17.6 & 0.3 \\
\hline $\mathrm{NCE}_{\nu}$ & 5.5 & 12.7 & 13.6 & 19.5 & 0.3 \\
\hline $\mathrm{NC} \pi^{0}{ }_{\nu}$ & 7.7 & 10.5 & 10.2 & 16.5 & 0.7 \\
\hline \multicolumn{6}{|c|}{ Antineutrino Mode } \\
\hline $\mathrm{CCQE}_{\bar{\nu}}$ & 5.6 & 18.4 & 9.3 & 21.4 & 0.3 \\
\hline $\mathrm{NCE}_{\bar{\nu}}$ & 4.7 & 16.0 & 19.7 & 27.8 & 0.4 \\
\hline $\mathrm{NC} \pi^{0}{ }_{\bar{\nu}}$ & 7.0 & 7.9 & 14.5 & 17.9 & 1 \\
\hline \multicolumn{6}{|l|}{ Off-Target } \\
\hline $\mathrm{CCQE}_{\mathrm{Off}}$ & 32.8 & 17.9 & 3.0 & 37.5 & 3.2 \\
\hline $\mathrm{NCE}_{\mathrm{Off}}$ & 25.9 & 7.7 & 7.8 & 28.2 & 2.6 \\
\hline $\mathrm{NC} \pi^{0}{ }_{\text {Off }}$ & 26.7 & 10.0 & 10.3 & 30.3 & 9 \\
\hline
\end{tabular}


gives the breakdown of the systematics on the total background prediction for all distributions considering bin-tobin correlations and no constraints. The total systematic uncertainty is the quadratic sum of the three categories given plus the uncertainties on the previous trigger activity cut and random trigger scaling. For CCQE and $\mathrm{NC} \pi^{0}$ the uncertainty on the previous trigger activity is zero and practically zero for the random trigger scaling. The reduction in the cross section and detector model uncertainties in $\mathrm{NCE}_{\text {Off }}$ compared to $\mathrm{NCE}_{\nu}$ comes from the increased percentage of beam-unrelated backgrounds. NCE has a lower total uncertainty in cross sections compared to CCQE because NCE (CCQE) is most uncertain at higher (lower) reconstructed 4-momentum transfer using the quasielastic assumption $Q_{\mathrm{QE}}^{2}$ [see Eqs. (3) and (4) for definitions], where there are less (more) events.

The total constrained uncertainty is calculated by considering that all nonsignal bins constrain the signal bins. For $\mathrm{NCE}_{\text {Off }}$ and $\mathrm{NC} \pi^{0}$ Off the total constrained uncertainties are $6.5 \%$ and $11.0 \%$ respectively. Statistical uncertainties are included in the total constrained calculation.

The shape-only uncertainty is $6.8 \%$ for $\mathrm{NC} \pi^{0 \text { Timing }}$ aff $2.3 \%$ for $\mathrm{NCE}_{\mathrm{Off}}^{\mathrm{Timing}}$, and is dominated by uncertainties in the detector model. The uncertainty in the instrumentation of the RWM and calibration of the simulation is small compared to the uncertainty from the detector model. When considering all nontiming distributions as constraining the timing distributions, the total constrained uncertainty is $4.1 \%$ for $\mathrm{NCE}_{\text {Off }}^{\text {Timing }}$ and $10.3 \%$ for $\mathrm{NC} \pi_{\text {Off }}^{0 \text { Timing }}$.

\section{Fit method}

Two different confidence level limits are extracted from the data: (i) full nucleon, and (ii) electron. Each fit methodology is described below.

\section{Full nucleon}

For this fit the signal distributions were $\mathrm{NCE}_{\text {Off }}$, $\mathrm{NC} \pi^{0}{ }_{\text {Off }}, \mathrm{NCE}_{\text {Off }}^{\text {Timing }}$, and $\mathrm{NC} \pi^{0 \text { Timing }}$. The CCQE, NCE, and $\mathrm{NC} \pi^{0}$ distributions from neutrino and antineutrino modes, as well as $\mathrm{CCQE}_{\text {Off }}$ were used to constrain the systematic uncertainties and predicted beam-related backgrounds in the signal channels. The CCQE and NCE distributions are fitted as functions of $Q_{\mathrm{QE}}^{2}$. The $Q_{\mathrm{QE}}^{2}$ for NCE is obtained via

$$
Q_{\mathrm{QE}}^{2}=2 m_{N} T_{N}^{\mathrm{reco}},
$$

where $m_{N}$ is the effective mass of the nucleon and $T_{N}^{\text {reco }}$ is the reconstructed kinetic energy of the nucleon recoil. The CCQE $Q_{\mathrm{QE}}^{2}$ is obtained via

$$
Q_{\mathrm{QE}}^{2}=-m_{\mu}^{2}+2 E_{\nu}^{\mathrm{QE}}\left(E_{\mu}-\sqrt{E_{\mu}^{2}-m_{\mu}^{2}} \cos \theta_{\mu}\right),
$$

where

$$
E_{\nu}^{\mathrm{QE}}=\frac{2 m_{n}^{\prime} E_{\mu}-\left[\left(m_{n}^{\prime}\right)^{2}+m_{\mu}^{2}-m_{p}^{2}\right]}{2\left[m_{n}^{\prime}-E_{\mu}+\sqrt{E_{\mu}^{2}-m_{\mu}^{2}} \cos \theta_{\mu}\right]},
$$

and $E_{\mu}=T_{\mu}^{\mathrm{reco}}+m_{\mu}$ is the total muon energy, and $m_{p}, m_{n}$ and $m_{\mu}$ are the masses of the proton, neutron and muon respectively. $m_{n}^{\prime}=m_{n}-E_{B}$ is the mass of the neutron minus the binding energy of carbon. A value of $34 \mathrm{MeV}$ is used for $E_{B}$. Both equations arise from kinematic calculations assuming the incident nucleon is at rest. The $\mathrm{NC} \pi^{0}$ distributions, on the other hand, are fitted as a function of reconstructed $\pi^{0}$ momentum $p_{\pi^{0}}$. As already stated the $\mathrm{CCQE}_{\text {Off }}$ timing distribution was used to calibrate the simulated $T_{\text {bunch }}$, so it was not included in the dark matter fit.

During the fit, one normalization nuisance parameter was used for each mode of running, constrained by the integral of the corresponding CCQE distribution. Two cross-section nuisance parameters were also used for each bin of the $\mathrm{NCE}\left(Q_{\mathrm{QE}}^{2}\right)$ and $\mathrm{NC} \pi^{0}\left(p_{\pi^{0}}\right)$ distributions: one for true neutrino and one for true antineutrino interactions. Neutrino and antineutrino interactions were considered separately because the neutrino/antineutrino interaction ratio is different between the three modes of running. In all, 23 nuisance parameters were used in the fit.

Fake data sets were used in generating the confidence level limits. Fake data was generated by randomly sampling around the predicted distributions with a Gaussian distribution containing the full event covariance matrix. When a nonzero amount of dark matter is assumed, the dark matter distribution is added on top of the predicted distributions before generating the fake data set.

When setting the confidence level limits the nuisance parameters were held fixed to make the neutrino, antineutrino, and $\mathrm{CCQE}_{\mathrm{Off}}$ distributions match the (fake) data being fitted. The nuisance parameters were held fixed because the signal distributions do not affect the resulting nuisance parameters. It was also determined, with a small slice in the dark matter parameter space, that allowing the nuisance parameters to float did not alter the resulting confidence level limit, but did increase the computation time significantly. The dark matter signal rate (controlled by a scaling factor) was floated during the confidence level limit calculation.

In off-target mode $990 \mathrm{CCQE}, 1461 \mathrm{NCE}$, and 148 $\mathrm{NC} \pi^{0}$ events were measured. After considering the constraints the predicted number of events is $1406 \pm 91$ and $135 \pm 15$ for $\mathrm{NCE}_{\text {Off }}$ and $\mathrm{NC} \pi^{0}$ off respectively. No significant excess was measured.

\section{Electron}

The signal distribution for this fit was defined as the events that pass $\nu$-e cuts with $\cos \theta_{e}>0.99$. The fit was a 
binned extended maximum-likelihood fit in three dimensions, $E_{\mathrm{vis}}^{e}, \cos \theta_{e}$, and bunch time, with a single nuisance parameter to control the overall normalization of predicted neutrino events. The region of $0.9<\cos \theta_{e}<0.99$ was the control region to constrain background events. Because of the well-defined control region, data from neutrino and antineutrino modes were not used to constrain the prediction. Two $\nu$-e events were measured in off-target mode. After constraining the $\nu$-e background the predicted number of events is $2.4 \pm 1.5$. In the signal region, zero events were measured with a constrained prediction of 0.4 events. Statistical error dominates the total error in the constrained prediction. No dark matter candidate events were measured.

Systematic uncertainties were not included in the fit as the predicted number of background events has a statistical relative uncertainty much greater than the predicted systematics, especially when considering that some of the systematic uncertainties are constrained by the controlled region. The normalization parameter is fixed during fitting so the data/fake data and null predictions are the same for the number of events in the control region. When generating the fake data for the electron analysis, each bin is assumed to be independent with an underlying Poisson distribution with a mean equal to the predicted plus dark matter distribution.

\section{Neutrino oscillation events in off-target mode}

As previously stated about $40 \%$ of the events that pass $\nu$ - $e$ cuts also pass neutrino oscillation cuts [23]. Figure 19 shows the $E_{\nu}^{\mathrm{QE}}$ distribution [Eq. (5) is used with the results

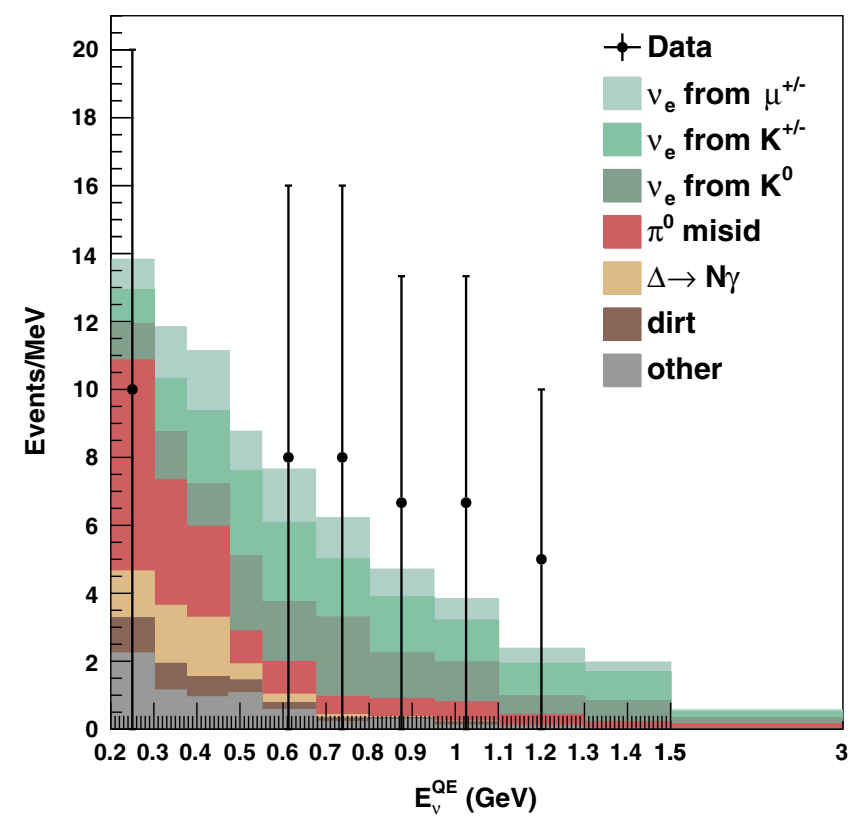

FIG. 19. The $E_{\nu}^{\mathrm{QE}}$ distribution for events that pass the $\nu_{e}$ oscillation cuts. Data comes from off-target mode. from the electron track fit and $E_{B}=0$ ] for off-target running. Simulation predicted 8.8 events assuming there are no oscillations. Six events were measured. All but one of the observed events were above $475 \mathrm{MeV}$. Implications of this data are discussed in Sec. VII B.

\section{CONFIDENCE LEVEL LIMITS ON LIGHT DARK MATTER THEORY}

A fixed-target dark matter Monte Carlo simulator, BDNMC, was used to simulate the energy and position distributions of the expected dark matter scattering signal in the MiniBooNE detector [29]. There are a number of production channels in fixed-target experiments, though often one will dominate for a given set of dark matter model parameters. For MiniBooNE, the decay of two pseudoscalar mesons, the $\pi^{0}$ and the $\eta$ were considered, as well as production through proton bremsstrahlung plus vector mixing up to $m_{V}=1 \mathrm{GeV} \mathrm{c} c^{-2}$. The parameter values and equations used in the simulation were given in Ref. [29].

The simulation loop begins by determining the maximum probability in the angular momentum distribution of each production channel, as it is not known analytically [29]. This maximum is used in an acceptance-rejection algorithm to sample the angular momentum distribution of each channel when generating dark matter trajectories. The total number of dark matter particles expected from each production channel is then calculated, and the output events are split between these channels according to their fraction of the total dark matter production rate.

For the case of pseudoscalar meson decays, meson 4-momenta and positions are generated in the MiniBooNE beam line by sampling an event list generated by the BooNEG4BEAm simulations; see Sec. III. For the case of proton bremsstrahlung, the dark matter is simulated to occur at the front of the beam dump.

The simulation attempts a given dark matter scattering event for each dark matter trajectory from the previous step found to intersect with the MiniBooNE detector. Possible interactions are elastic-nucleon $(0 \pi)$, elastic-electron, and inelastic-nucleon producing a single pion $\left(1 \pi^{0}\right.$ if a $\pi^{0}$ is produced, and $1 \pi^{ \pm}$if a $\pi^{ \pm}$is produced). The neutrino detector simulation, discussed in Sec. IVA, was used to simulate the response of the detector. This simulation used neutrino events generated by NUANCE and contained the nuclear model and all final-state interactions. We define the weight of each neutrino simulated event as the ratio $N_{\chi}(\omega) / N_{\nu}(\omega)$, where $N(\omega)$ is the number of true interactions as a function of energy transfer $\omega$ that generated the simulated event. $N_{\chi}(\omega)$ is the number of interactions predicted by BDNMC and $N_{\nu}(\omega)$ is the number of true interactions predicted by NUANCE. Since $N_{\nu}(\omega)$ comes from the true distribution, which contains no nuclear model nor final-state interactions, the resulting reconstructed dark 

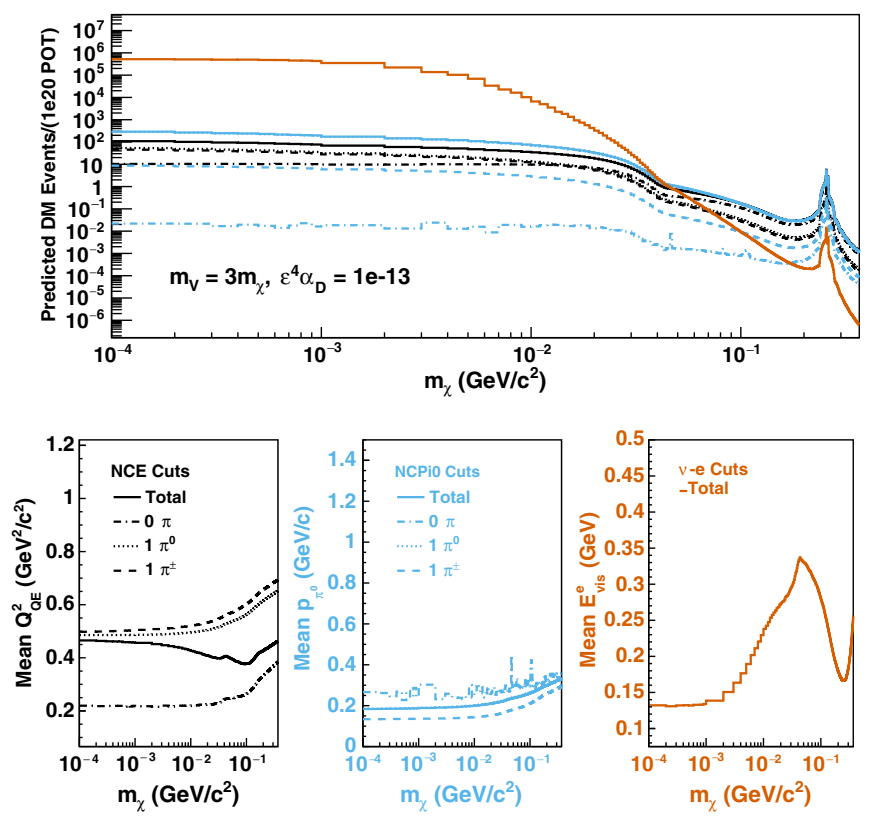

FIG. 20. (Top) The integral number of events that pass NCE, $\mathrm{NC} \pi^{0}$, and $\nu$ - $e$ selection cuts as a function of $m_{\chi}$. Predictions for the total, $0 \pi, 1 \pi^{0}$, and $1 \pi^{ \pm}$are given by solid, dash-dotted, dotted, and short-dashed lines respectively. The colors of the lines correspond with the bottom figures. (Bottom left) The mean $Q_{\mathrm{QE}}^{2}$ distribution for NCE selection cuts. (Bottom middle) The mean $p_{\pi}^{0}$ distribution for $\mathrm{NC} \pi^{0}$ selection cuts. (Bottom right) The mean $E_{\mathrm{vis}}^{e}$ distribution for $\nu$-e selection cuts. All plots are functions of $m_{\chi}$ with $m_{V}=3 m_{\chi}$ and $\epsilon^{4} \alpha_{D}=1 \times 10^{-13}$.

matter distribution contains the nuclear model and the model of final-state interactions that are in NUANCE.

Figure 20 shows the number of events for $\chi$ scattering in the detector as well as the mean reconstructed observables for $m_{V}=3 m_{\chi}$ and $\epsilon^{4} \alpha_{D}=1 \times 10^{-13}$. At low masses the $1 \pi$ dominates over $0 \pi$ in overall rate for nucleon interactions. The $1 \pi$ production dominates the NCE distribution at higher $Q_{\mathrm{QE}}^{2}$. Because of the separation of where $1 \pi$ and

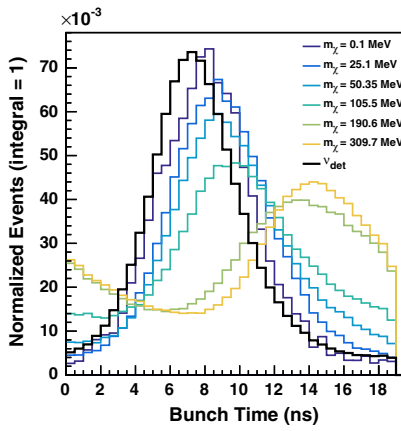

(a) $\mathrm{NCE}$

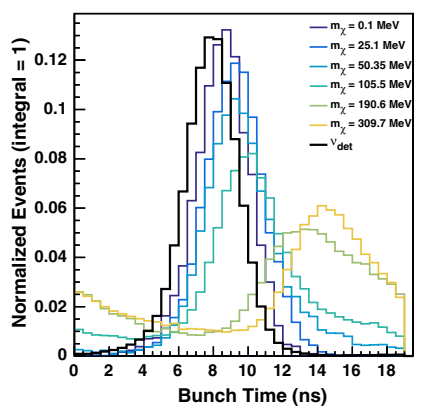

(b) $\mathrm{NC} \pi^{0}$
FIG. 21. Comparison between signal interactions that pass (a) NCE and (b) $\mathrm{NC} \pi^{0}$ cuts to different dark matter distributions as a function of bunch time. The neutrino interactions are for the neutrino mode while dark matter is for the off-target mode (see text).
$0 \pi$ production dominate the NCE distribution, and the efficiency of the NCE selection cuts, NCE provides significant constraint, along with $\mathrm{NC} \pi^{0}$, on the low-mass region. Dark matter scattering off electrons is predicted to dominate the overall rate at $m_{\chi}<0.4 \mathrm{GeV} c^{-2}$.

Figure 21 compares the bunch-time distribution for various combinations of $m_{V}$ and $m_{\chi}$ to the neutrino

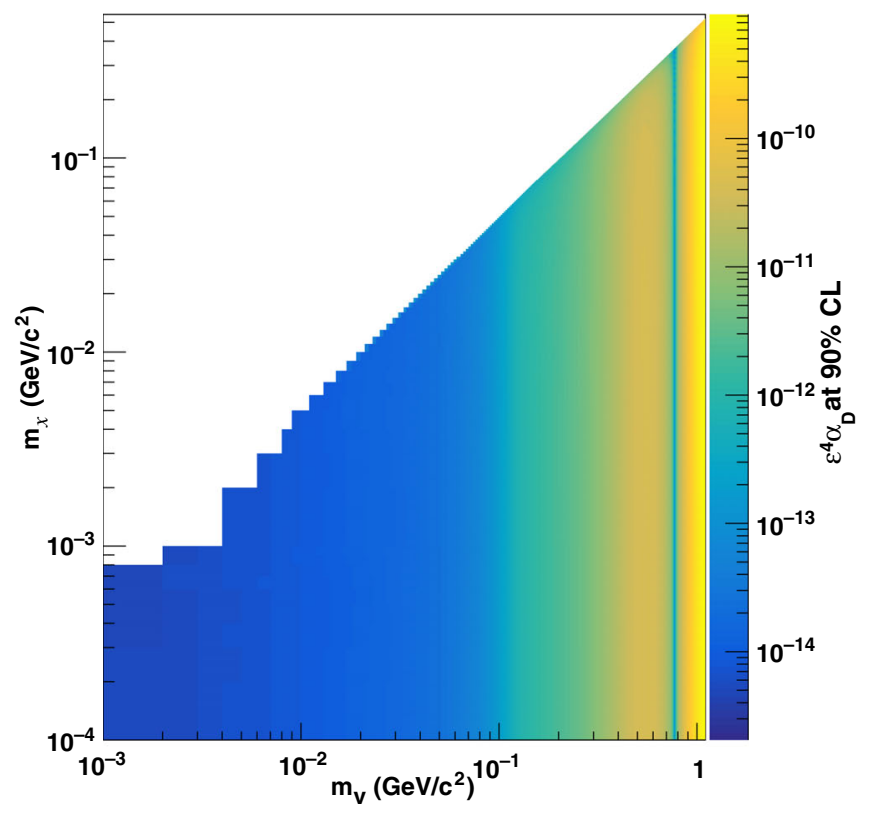

(a) Full Nucleon + Timing

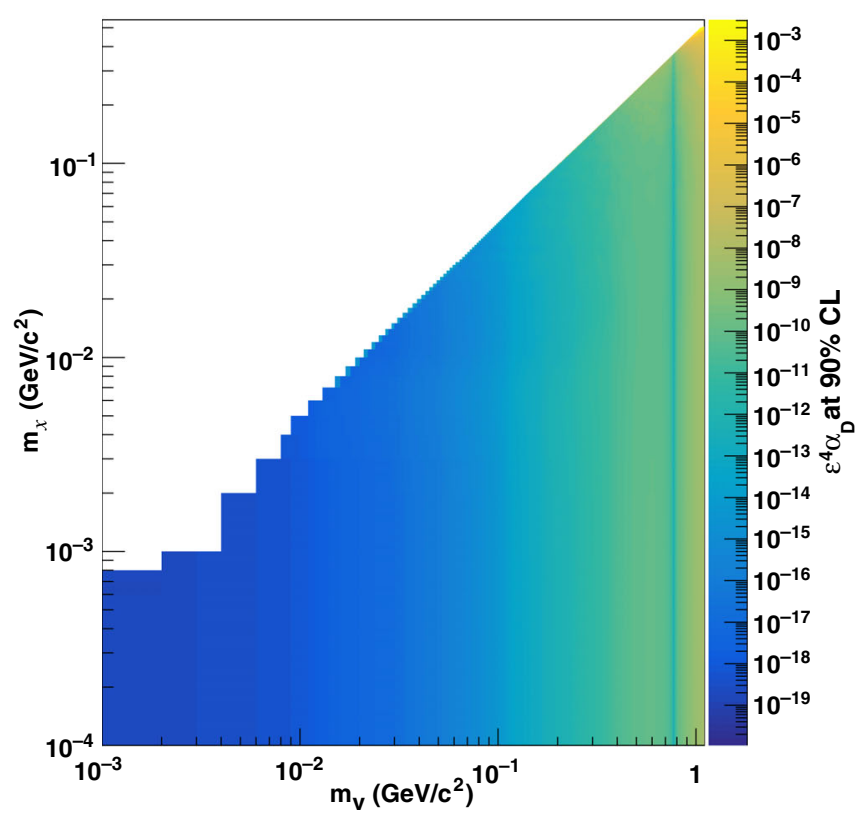

(b) Electron + Timing

FIG. 22. The $90 \%$ confidence level limit for (a) full nucleon with timing and (b) electron with timing on $\epsilon^{4} \alpha_{D}$ for various combinations of $m_{V}$ and $m_{\chi}$ using the vector portal dark matter model. 
distribution used for the candidate signal events that pass $\mathrm{NCE}$ and $\mathrm{NC} \pi^{0}$ selection cuts. The neutrino distribution is the predicted distribution for neutrino-mode running, while the dark matter distributions are for off-target mode. The difference between the neutrino distribution and that of the lightest dark matter mass represents the difference between neutrino-mode and off-target running, which is consistent with the average time difference between the dark matter and the neutrino to reach the steel beam dump. The sensitivity for heavier dark matter masses is improved when using timing.

Using the results from BDNMC and the frequentist confidence level method developed for the MiniBooNE oscillation analysis [21], 90\% confidence level limits were calculated for different combinations of $m_{V}$ and $m_{\chi}$ as a function of $\epsilon^{4} \alpha_{D}$. The frequentist approach used fake data and various fits to fake data to generate the effective d.o.f. given a predicted signal. Each combination of $m_{V}$ and $m_{\chi}$ was treated independently, and because only on-shell decay was considered (see Sec. II), $\epsilon^{4} \alpha_{D}$ controls only the normalization of the predicted dark matter signal. Figure 22 gives the $90 \%$ confidence level limits on $\epsilon^{4} \alpha_{D}$ as a function of $m_{V}$ and $m_{\chi}$ for both the full nucleon and electron fits when including timing. The best limit, in the tested parameter space, was set at $m_{V}=0.3 \mathrm{MeV} \mathrm{c}^{-2}, m_{\chi}=$ $0.1 \mathrm{MeV}^{-2}$ with $\epsilon^{4} \alpha_{D}=3.9 \times 10^{-15}$ for the full nucleon fit and $m_{V}=0.5 \mathrm{MeV}^{-2}, m_{\chi}=0.2 \mathrm{MeV} \mathrm{c}^{-2}$ with $\epsilon^{4} \alpha_{D}=1.3 \times 10^{-19}$ for the electron fit.

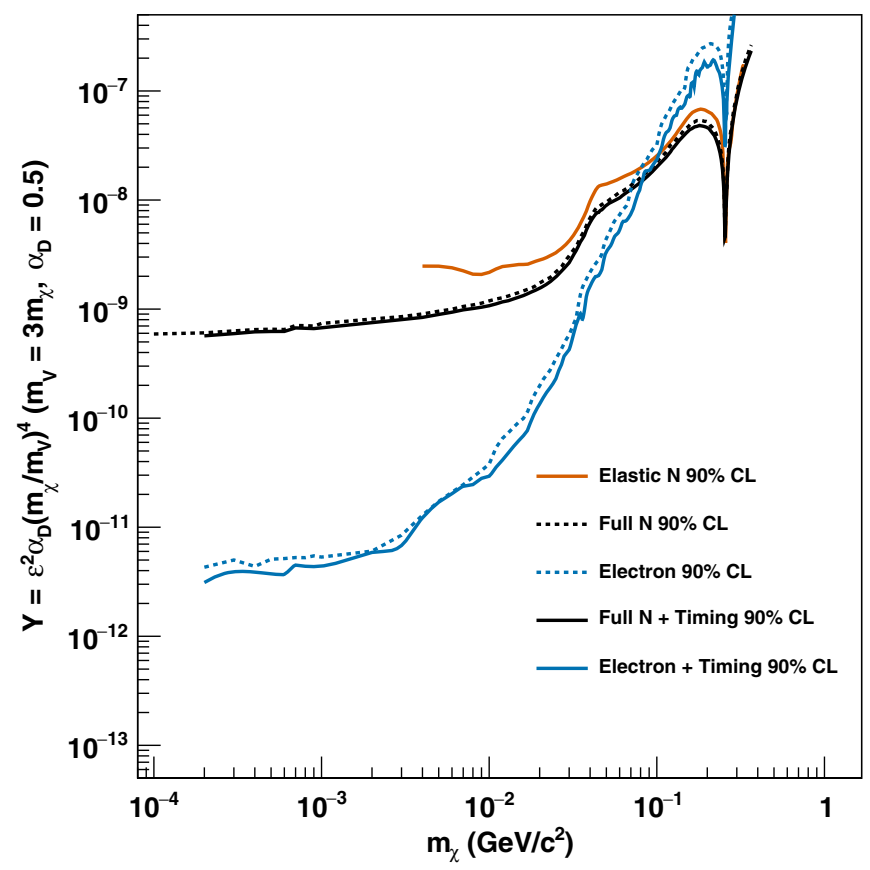

FIG. 23. Comparing the full nucleon and electron confidence level results to the elastic nucleon results from Ref. [20]. Also shown is the result when including timing (solid lines) compared to that when not including the timing (dashed lines).
Figure 23 compares the confidence level results in this paper to the elastic-nucleon results [20] for the dark matter parameter slice $m_{V}=3 m_{\chi}$ and $\alpha_{D}=0.5$, where $Y=$ $\epsilon^{2} \alpha_{D}\left(m_{\chi} / m_{V}\right)^{4}$ is a dimensionless parameter that controls the dark matter annihilation cross section and in turn the thermal relic abundance. Also shown are the confidence level limits when timing is not included. Including the $1 \pi \chi$ interaction channels improves the confidence level from Ref. [20] at low masses while including timing improves the confidence level limits at high masses in $\epsilon^{4} \alpha_{D}$ up to a factor of 1.5 for the full nucleon fit and 4.7 for the electron fit. For the variable $Y$ this corresponds to improvements in the confidence level limits by 1.2 and 2.2 for the full nucleon and electron fits respectively. The electron fit gives more restrictive limits at lower masses compared to the full nucleon fit.

\section{RESULTS AND DISCUSSION}

The following is a discussion on the implications of the results presented above. A comparison of the full nucleon and electron dark matter analyses with current limits will be discussed, followed by the implications of not seeing an excess in the neutrino oscillation sample. The section will conclude with the implications of running with a proposed dedicated "beam-dump" target.

\section{A. Limits on sub-GeV dark matter}

MiniBooNE has improved upon the results published in Ref. [20] through dedicated searches for $\pi^{0}$ production and elastic scattering initiated by dark matter particles produced in a proton beam dump. The dark matter search built upon a rich history of cross section and oscillation analyses already published by the MiniBooNE Collaboration. The full nucleon dark matter analysis leveraged neutrino and antineutrino data sets, as well as the $\mathrm{CCQE}_{\text {Off }}$ distribution to constrain systematic uncertainties. Both the full nucleon and electron analyses utilized the use of the BNB bunch structure to set stronger limits on heavier $m_{\chi}$.

Figures 24(a), 25(a), and 25(b) show three example projections of the limits in Fig. 22 to the $m_{\chi}-Y$ plane. The chosen projections are standard but are not the only ones possible. The differences between the three slices are due to different assumptions about $\alpha_{D}$ and the relationship between $m_{\chi}$ and $m_{V}$.

Two different relationships between $m_{\chi}$ and $m_{V}$ are shown to demonstrate how the contours in the $m_{\chi}-Y$ plane change as a function of model parameters. The relic density contour (green) indicates the $m_{\chi}, Y$ values where the model with a complex scalar dark matter particle predicts a dark matter abundance that is in accord with observations. As the ratio $m_{V} / m_{\chi}$ increases, the constraints on the thermal relic target become more stringent and can be ruled out over the full parameter space; see Fig. 25(b). Furthermore, as the ratio $m_{V} / m_{\chi}$ increases, other kinds of dark matter 


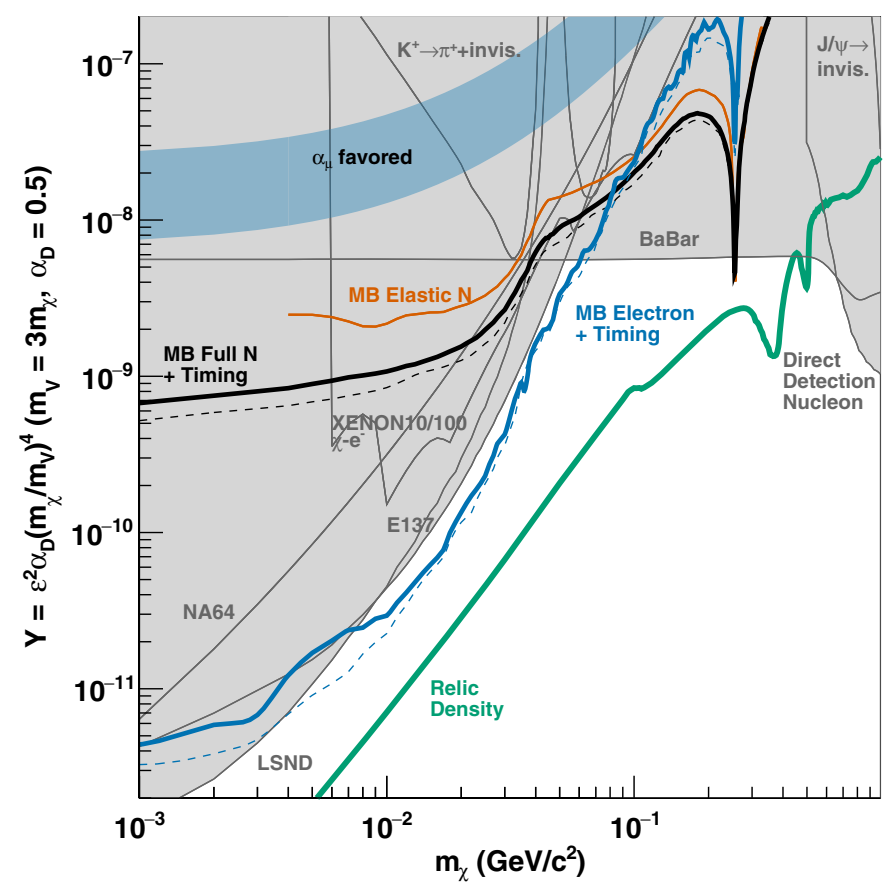

(a) vector portal

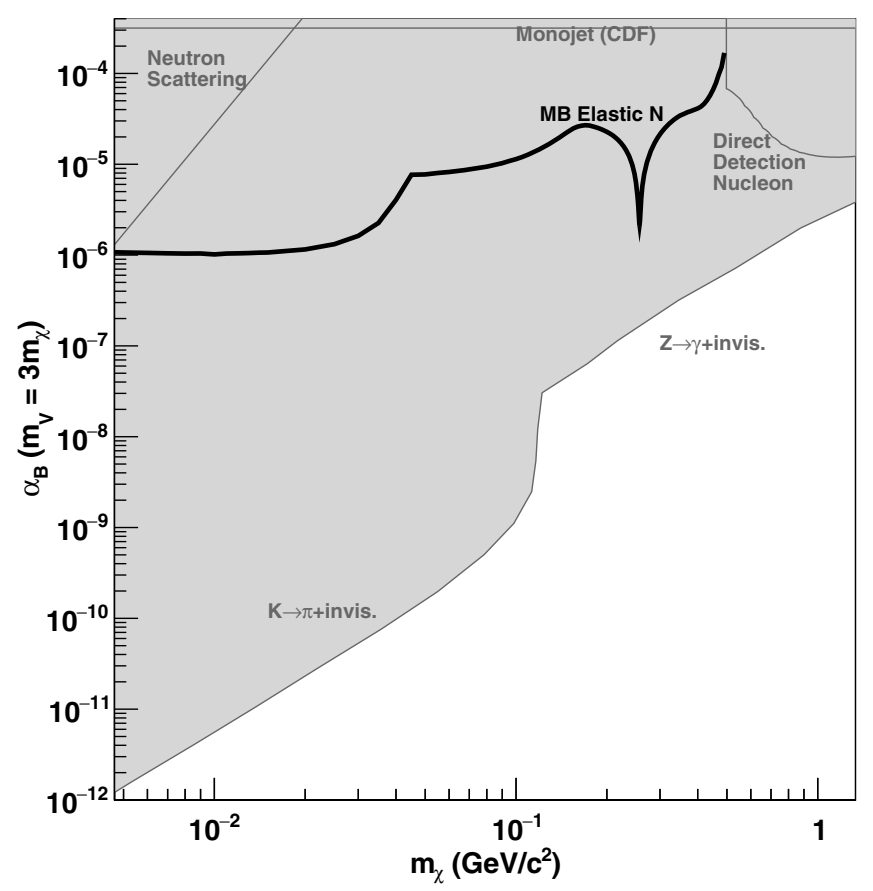

(b) leptophobic

FIG. 24. Comparison of the MiniBooNE confidence level limits (solid lines), and sensitivities (dashed lines) to other experiments for (a) $Y$ as a function of $m_{\chi}$ assuming $\alpha_{D}=0.5$ and $m_{V}=3 m_{\chi}$ and (b) in the leptophobic dark matter model with $m_{V}=3 m_{\chi}$. An explanation of vector portal limits lines was given in Refs. [9,29,36-38]. An explanation of the leptophobic limit lines was given in Refs. [8,34,35].

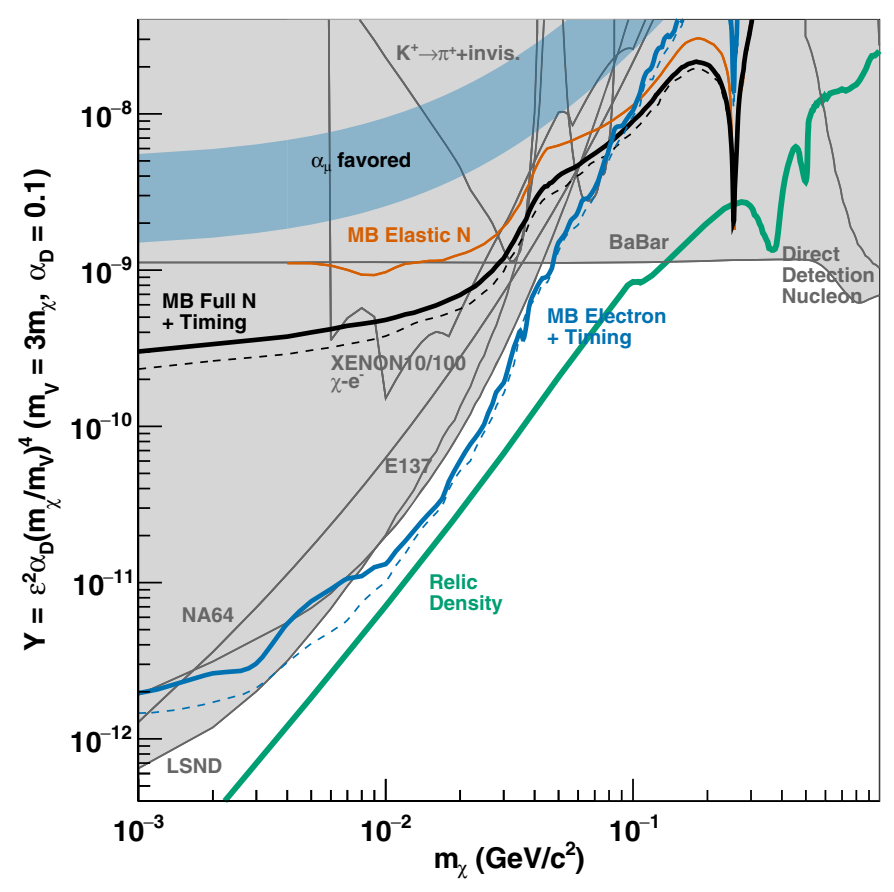

(a) vector portal $\left(\alpha_{D}=0.1, m_{V}=3 m_{\chi}\right)$

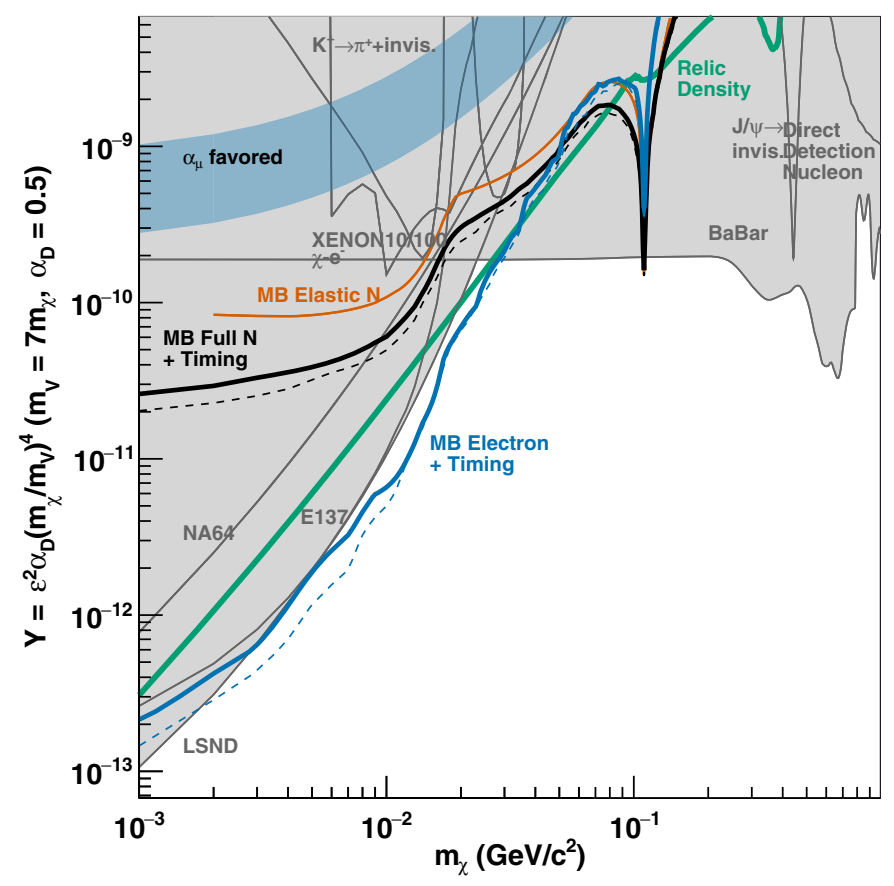

(b) vector portal $\left(\alpha_{D}=0.5, m_{V}=7 m_{\chi}\right)$

FIG. 25. $90 \%$ confidence level in the vector portal dark matter model with (a) $Y$ as a function of $m_{\chi}$ assuming $\alpha_{D}=0.1$ and $m_{V}=3 m_{\chi}$ and (b) $\alpha_{D}=0.1$ and $m_{V}=7 m_{\chi}$. An explanation of the limit lines was given in Refs. [9,29,36-38]. 
scenarios, e.g., asymmetric fermionic dark matter, can be probed by MiniBooNE and other current experiments [59]. At smaller $m_{V} / m_{\chi}$ ratios there is still a wide region of parameter space in the complex scalar dark matter model that can satisfy the relic density requirement; see Figs. 24(a) and 25(a).

For the vector portal model, MiniBooNE excludes the muon $g-2$ favored region, and some regions where this model satisfies the relic density in the parameter space tested. MiniBooNE also excludes previously untested parameter space, especially in the electron channel. For the leptophobic dark matter scenario, inelastic neutral pion production has not been studied in the literature. Therefore, the nucleon elastic results from Ref. [20] were used to place conservative limits on this scenario. The result can be found in Fig. 24(b).

\section{B. MiniBooNE neutrino oscillation excess}

MiniBooNE has recently doubled the amount of neutrino-mode POT [23]. The reported neutrino plus antineutrino oscillation excess is $460.5 \pm 99.0$ for a combined $24.11 \times 10^{20}$ POT. If this excess were due to a process that is occurring in the beam dump, such as dark matter production, instead of neutrino-related processes, the predicted excess would scale with the amount of POT collected.

An example process that would scale solely by POT would be the production of a dark mediator through neutral meson decay or proton bremsstrahlung which would then decay into two dark matter particles. One of the dark matter particles would then decay in the detector producing a lepton-antilepton pair with low invariant mass. A potential dark matter model that could be extended to fit such a description can be found in Refs. [17,60].

The predicted off-target excess, under this assumption, is $35.5 \pm 7.6$, whereas the measured excess is -2.8 events integrated over $200 \leq E_{\nu}^{\mathrm{QE}}<1250 \mathrm{MeV}$; see Sec. V C 3 . Assuming Gaussian errors, the measured off-target sample of events that pass oscillation cuts is inconsistent, at $4.6 \sigma$, with a process that predicts that all of the oscillation excess scales with the collected POT independent of the beam configuration.

\section{Proposed dedicated "beam-dump" target}

MiniBooNE has shown that a neutrino experiment can search for fixed-target accelerator-produced dark matter scattering for different production and interaction channels. Most of the neutrino backgrounds came from proton interactions in the air and scraping of the target. To further reduce the neutrino background a dedicated "beam-dump" target is needed. A simulation of a steel beam dump target positioned where the neutrino target/horn are located, effectively removing the decay pipe, indicates the decrease of the $\mathrm{CCQE}_{\text {Off }}$ event rate by a factor of 20 . The $\mathrm{NC} \pi^{0}$ and $\nu$-e sensitivities would increase the most with this reduction in the beam-related backgrounds. For example, a total of five events are predicted to pass $\mathrm{NC} \pi^{0}$ selection cuts for $1.86 \times 10^{20}$ POT compared to the 148 measured in this analysis. The reduction for $\mathrm{NC} \pi^{0}$ is larger than NCE or CCQE because more energetic neutrinos are required to generate $\mathrm{NC} \pi^{0}$ events.

A dedicated "beam-dump" target would also decisively test theories that predict the oscillation excess scales as POT. With a dedicated "beam-dump" target almost no events are expected to pass oscillation cuts. An upgrade is being considered that would add a secondary "beam-dump" target to the BNB [61]. The addition of the second target would allow simultaneous running, on a pulse-by-pulse basis, of protons hitting the neutrino and "beam-dump" targets. This would increase the physics output of the ShortBaseline Neutrino Program [62] at Fermilab.

\section{ACKNOWLEDGMENTS}

This work was supported by the U.S. Department of Energy (DOE); the U.S. National Science Foundation; Los Alamos National Laboratory; the Science and Technology Facilities Council, UK; and Consejo Nacional de Ciencia y Tecnologa, Mexico. We thank the Fermilab Accelerator Division for the work to reconfigure, operate, and understand the off-target beam. Fermilab is operated by Fermi Research Alliance, LLC under Contract No. De-AC0207CH11359 with the US DOE. We also thank Los Alamos National Laboratory for Los Alamos Rsearch and Development (LDRD) funding.
[1] B. Batell, M. Pospelov, and A. Ritz, Phys. Rev. D 80, 095024 (2009).

[2] P. deNiverville, M. Pospelov, and A. Ritz, Phys. Rev. D 84, 075020 (2011).

[3] P. deNiverville, D. McKeen, and A. Ritz, Phys. Rev. D 86, 035022 (2012).
[4] R. Dharmapalan et al. (MiniBooNE Collaboration), arXiv: 1211.2258.

[5] E. Izaguirre, G. Krnjaic, P. Schuster, and N. Toro, Phys. Rev. D 88, 114015 (2013).

[6] E. Izaguirre, G. Krnjaic, P. Schuster, and N. Toro, Phys. Rev. D 91, 094026 (2015). 
[7] E. Izaguirre, G. Krnjaic, P. Schuster, and N. Toro, Phys. Rev. D 90, 014052 (2014).

[8] B. Batell, P. deNiverville, D. McKeen, M. Pospelov, and A. Ritz, Phys. Rev. D 90, 115014 (2014).

[9] B. Batell, R. Essig, and Z. Surujon, Phys. Rev. Lett. 113, 171802 (2014).

[10] B. A. Dobrescu and C. Frugiuele, J. High Energy Phys. 02 (2015) 019.

[11] D. E. Soper, M. Spannowsky, C. J. Wallace, and T. M. P. Tait, Phys. Rev. D 90, 115005 (2014).

[12] Y. Kahn, G. Krnjaic, J. Thaler, and M. Toups, Phys. Rev. D 91, 055006 (2015).

[13] E. Izaguirre, G. Krnjaic, P. Schuster, and N. Toro, Phys. Rev. Lett. 115, 251301 (2015).

[14] P. deNiverville, M. Pospelov, and A. Ritz, Phys. Rev. D 92 , 095005 (2015).

[15] E. Izaguirre, G. Krnjaic, and M. Pospelov, Phys. Rev. D 92, 095014 (2015).

[16] P. Coloma, B. A. Dobrescu, C. Frugiuele, and R. Harnik, J. High Energy Phys. 04 (2016) 047.

[17] E. Izaguirre, Y. Kahn, G. Krnjaic, and M. Moschella, Phys. Rev. D 96, 055007 (2017).

[18] C. Frugiuele, Phys. Rev. D 96, 015029 (2017).

[19] G. Magill, R. Plestid, M. Pospelov, and Y.-D. Tsai, arXiv:1806.03310.

[20] A. A. Aguilar-Arevalo et al. (MiniBooNE Collaboration), Phys. Rev. Lett. 118, 221803 (2017).

[21] A. A. Aguilar-Arevalo et al. (MiniBooNE Collaboration), Phys. Rev. Lett. 110, 161801 (2013).

[22] A. A. Aguilar-Arevalo et al. (MiniBooNE Collaboration), Phys. Rev. D 81, 092005 (2010).

[23] A. A. Aguilar-Arevalo et al. (MiniBooNE Collaboration), arXiv:1805.12028.

[24] C. Boehm and P. Fayet, Nucl. Phys. B683, 219 (2004).

[25] P. Fayet, Phys. Rev. D 70, 023514 (2004).

[26] M. Pospelov, A. Ritz, and M. B. Voloshin, Phys. Lett. B 662, 53 (2008).

[27] N. Arkani-Hamed, D. P. Finkbeiner, T. R. Slatyer, and N. Weiner, Phys. Rev. D 79, 015014 (2009).

[28] B. Holdom, Phys. Lett. B 166, 196 (1986).

[29] P. deNiverville, C.-Y. Chen, M. Pospelov, and A. Ritz, Phys. Rev. D 95, 035006 (2017).

[30] P. A. R. Ade et al. (Planck Collaboration), Astron. Astrophys. 594, A13 (2016).

[31] P. Fayet, Nucl. Phys. B347, 743 (1990).

[32] P. Fayet, Eur. Phys. J. C 77, 53 (2017).

[33] B. A. Dobrescu and C. Frugiuele, Phys. Rev. Lett. 113, 061801 (2014).

[34] J. A. Dror, R. Lasenby, and M. Pospelov, Phys. Rev. Lett. 119, 141803 (2017).

[35] J. A. Dror, R. Lasenby, and M. Pospelov, Phys. Rev. D 96, 075036 (2017).
[36] D. Banerjee et al. (NA64 Collaboration), Phys. Rev. D 97, 072002 (2018).

[37] R. Essig, T. Volansky, and T.-T. Yu, Phys. Rev. D 96, 043017 (2017).

[38] J. P. Lees et al. (BABAR Collaboration), Phys. Rev. Lett. 113, 201801 (2014).

[39] S. Agostinelli et al. (GEANT4 Collaboration), Nucl. Instrum. Methods A 506, 250 (2003).

[40] A. Aguilar-Arevalo et al. (MiniBooNE Collaboration), Phys. Rev. D 79, 072002 (2009).

[41] R. T. Thornton, Search for light dark matter produced in a proton beam dump, Ph.D. thesis, Indiana University, 2017.

[42] V. Singh, Phys. Rev. Lett. 28, 859 (1972).

[43] E. Amaldi et al., Nucl. Phys. B158, 1 (1979).

[44] K. Jaeger, J. Campbell, G. Charlton, D. Swanson, C. Fu, H. A. Rubin, R. G. Glasser, D. Koetke, and J. Whitmore, Phys. Rev. D 11, 1756 (1975).

[45] T. Sjostrand, S. Mrenna, and P. Z. Skands, J. High Energy Phys. 05 (2006) 026.

[46] B. Fellenz and J. L. Crisp, AIP Conf. Proc. 451, 446 (1998).

[47] M. Backfish, MiniBooNE resistive wall current monitor, Fermi National Accelerator Laboratory (FNAL) Technical Report FERMILAB-TM-2556-AD, 2013.

[48] A. A. Aguilar-Arevalo et al. (MiniBooNE Collaboration), Nucl. Instrum. Methods A 599, 28 (2009).

[49] C. Athanassopoulos et al. (LSND Collaboration), Nucl. Instrum. Methods A 388, 149 (1997).

[50] D. Casper, Nucl. Phys. B Proc. Suppl. 112, 161 (2002).

[51] A. A. Aguilar-Arevalo et al. (MiniBooNE Collaboration), Phys. Rev. D 82, 092005 (2010).

[52] A. A. Aguilar-Arevalo et al. (MiniBooNE Collaboration), Phys. Rev. D 81, 013005 (2010).

[53] D. Rein and L. M. Sehgal, Ann. Phys. (N.Y.) 133, 79 (1981).

[54] D. Rein and L. M. Sehgal, Nucl. Phys. B223, 29 (1983).

[55] R. Brun, F. Bruyant, M. Maire, A. C. McPherson, and P. Zanarini, GEANT3, CERN Reprot No. CERN-DD-EE-84-1, (1987).

[56] A. Aguilar-Arevalo et al. (MiniBooNE Collaboration), Phys. Rev. D 91, 012004 (2015).

[57] R. B. Patterson, E. M. Laird, Y. Liu, P. D. Meyers, I. Stancu, and H. A. Tanaka, Nucl. Instrum. Methods A 608, 206 (2009).

[58] R. Patterson, A search for muon neutrino to electron neutrino oscillations at $\Delta m^{2}>1 \mathrm{eV}^{2}$, Ph.D. thesis, Princeton University, 2007.

[59] M. Battaglieri et al., arXiv:1707.04591.

[60] J. R. Jordan, Y. Kahn, G. Krnjaic, M. Moschella, and J. Spitz, Phys. Rev. D 98, 075020 (2018).

[61] See talk by R. G. Van de Water, https://indico.fnal.gov/ event/15726/session/3/material/0/0.pdf.

[62] M. Antonello et al. (LAr1-ND, ICARUS-WA104, and MicroBooNE Collaborations), arXiv:1503.01520. 